\title{
Research Paper \\ Predictive Role of Morningness-Eveningness Personality, Cognitive Flexibility and Cognitive Emotion Regulation in Marital Satisfaction in Middle-Aged Women
}

\author{
${ }^{*}$ Hossein Shareh ${ }^{1} \odot$, Maryam Eshaghi Sani ${ }^{2} \odot$
}

1. PhD., Associate Professor, Department of Educational Sciences, Faculty of Literature and Humanities, Hakim Sabzevari University, Sabzevar, Iran. 2. MSc., Department of Psychology, Faculty of Humanities, Neyshabur Branch, Islamic Azad University, Neyshabur, Iran.

\begin{tabular}{|c|c|}
\hline $\begin{array}{l}\text { Use your device to scan } \\
\text { and read the article online }\end{array}$ & detion Shareh H, Eshaghi Sani M. Predictive Role of Morningness-Eveningness Personality, Cognitive Flexibility and Cog- \\
\hline 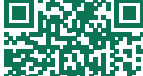 & $\begin{array}{l}\text { nitive Emotion Regulation in Marital Satisfaction in Middle-Aged Women. Iranian Journal of Psychiatry and Clinical Psychology. } \\
\text { 2019; 24(4):384-399. http://dx.doi.org/10.32598/ijpcp.24.4.384 }\end{array}$ \\
\hline (1) & d sl": http://dx.doi.org/10.32598/ijpcp.24.4.384 \\
\hline
\end{tabular}

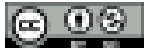

Received: 13 Oct 2017 Accepted: 31 Jul 2018 Available Online: 01 Jan 2019

Keywords: Marital satisfaction Flexibility, Cognition, Emotion, Morningnesseveningness

\section{A B S T R A C T}

Objectives Recent evidence suggests the effective role of physiological, cognitive and emotional factors in the quality of couple relationships. The purpose of this study was to study the relationship between morningness-eveningness personality, Cognitive Flexibility (CF) and Cognitive Rmotion Regulation (CER) with marital satisfaction in middle-aged women.

Methods The research method was correlational. Statistical population included all middle-aged married women (40-60 years old) living in Mashhad City, Iran. District 1 was randomly selected as the sample area and 211 women in this district were selected using systematic sampling method. Each participant completed Enrich marital satisfaction scale, cognitive flexibility inventory, morningness-eveningness questionnaire and short form of cognitive emotion regulation questionnaire. The obtained data were analyzed by Pearson correlation and multivariate regression.

Results Multivariate regression results indicated that alternatives option perception, control perception and total score of cognitive flexibility positively predicts marital satisfaction $(P<0.001)$. Furthermore, the role of a morningness-eveningness personality in predicting marital satisfaction was significant in a way that high scores in morningness matched with high scores in marital satisfaction $(\mathrm{P}<0.001)$. The results also indicated that self-blame, rumination, catastrophizing, and blame of others negatively $(P<0.001)$, acceptance and perspective-taking positively predict marital satisfaction $(P<0.05)$.

Conclusion The findings of this study, while expanding the literature of the research, highlight the physiological, cognitive and emotional characteristics of marital satisfaction in enrichment programs and premarital counseling.

\section{Extended Abstract}

\section{Introduction}

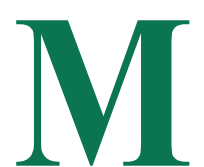

arital satisfaction is defined as the individuals' satisfaction with their marital life. New evidence suggests the effective role of physiological, cognitive, and emotional factors in the quality of the relationship between couples. One of the biological variables that can affect couples' compatibility is sleep-wake cycle and one of the most important individual differences is morningnesseveningness personality.

Evidence shows that morning people obtain higher scores in memory and attention. Morningness is positively related to life satisfaction and quality of life. On the other hand, eveningness is positively associated with depression, low

* Corresponding Author: Hossein Shareh, PhD.

Address: Department of Educational Sciences, Faculty of Literature and Humanities, Hakim Sabzevari University, Sabzevar, Iran. Tel: +98 (51) 44410104

E-mail: h.shareh@hsu.ac.ir 
sleep quality, undesirable personality profile, and healthdamaging behaviors. Based on what was discussed, morningness-eveningness preferences can partly predict personality type, quality of life and life satisfaction and thus marital satisfaction. Another variable that may be related to the rate of marital satisfaction is couples' cognitive function. Cognitive flexibility is one of the most fundamental dimensions of cognitive functions and is among the most important elements of executive functions, applied to the individual's ability to change thoughts, actions, and strategies in response to changes in circumstances. Flexible cognition requires the activation and modification of cognitive processes in response to calls for changes in doing an activity or even changes in the instruction for doing an activity.

People with the ability to think flexibly use alternative justifications, positively rebuild their intellectual framework, accept challenging situations or stressful events and are psychologically more resilient compared to those who have less flexibility. In addition to physiological and cognitive variables, one of the most important deficiencies that has a profound effect on the quality of communication patterns of individuals includes emotion regulation strategies. Since psychological adjustment largely depends on emotion regulation, this ability can affect couples' marital satisfaction. The present study aims to investigate the relationship between morningness-eveningness personality, cognitive flexibility and cognitive emotion regulation with marital satisfaction in married middle-aged women.

\section{Method}

The current study was a correlational research. The statistical population comprised all married middle-aged women ( $40-60$ years old) living in Mashhad City, Iran. District 1 was randomly selected as the sample area and from this district, 211 women were chosen through systematic sampling method. The research inclusion criteria consisted of the following: female gender, age between 40 and 60 years, living in Mashhad at least for the past two years and having at least a diploma.

The exclusion criteria were as follows: widows and divorced people, complete cessation of sexual relationship with the spouse, having extramarital relationships, having serious suicidal thoughts, having a history of admission to psychiatric hospital, suffering from the symptoms of psychotic disorders and drug addiction. The participants completed enrich marital satisfaction scale, cognitive flexibility inventory, morningness-eveningness questionnaire and short form of cognitive emotion regulation questionnaire. The obtained data were analyzed using Pearson correlation test and multivariate regression analysis.

\section{Results}

The relationship between psychological flexibility and marital satisfaction $(\mathrm{r}=0.26)$ and the relationship between all the subscales of cognitive flexibility and marital satisfaction were significant and positive $(\mathrm{P}<0.05)$. Multivariate regression results revealed that the subscales of perception of alternative options, perception of controllability, and total score of cognitive flexibility are able to positively predict marital satisfaction $(\mathrm{P}<0.05)$. Moreover, the role of morningness-eveningness personality was significant in predicting marital satisfaction, and high morningness was associated with high marital satisfaction $(\mathrm{r}=42, \mathrm{P}<0.01)$. Additionally, the results about emotion regulation components demonstrated that self-blame, rumination, catastrophizing and blame of others negatively predict marital satisfaction, and acceptance and perspective-taking positively predict marital satisfaction $(\mathrm{P}<0.05)$.

\section{Discussion}

Results of the present study indicate a significant and positive relationship between marital satisfaction and psychological flexibility. Cognitive flexibility is one of the dimensions of executive functions which refers to the ability to change the actions and thoughts in accordance with environmental demands. In other words, when a person uses cognitive strategies to change behavior and adapt to the environment, he enjoys flexible cognition. The relationship between couples also includes various complex and challenging situations which require the use of a flexible cognitive pattern.

In the event of conflicts and disagreements, successful couples benefit from negotiation and effective emotional and cognitive strategies. These strategies, while ending the conflicts and disagreements, bring couples emotionally closer together and cause them to better perceive each other's support. Accordingly, high cognitive flexibility is expected to be accompanied by marital satisfaction. Morning people compared to evening people are more optimistic and are emotionally stable, serious, and reliable. Morningness personality enjoys more cognitive processes, leading to more adaptation and adjustment with the environment.

All of these facts, therefore, lead to greater satisfaction with relationships, especially the relationships between couples. Cognitive emotion regulation is applied with the purpose of emotion management to increase adjustment and compatibility and is part of adaptation strategies that are associated with experience and treatment of emotional and physical discomfort. Accordingly, it is expected that high capability in cognitive emotion regulation lead to 
greater marital adjustment between couples and consequently increased satisfaction.

Cognitive emotion regulation plays a fundamental role in various normal and abnormal processes and is one of the important components of the coping process against negative stimuli and unpleasant emotional experiences. Therefore, low emotion regulation skill is associated with poor coping and thus experience of negative emotions and these emotions affect the quality of the relationship between couples and can reduce marital satisfaction. With regard to the use of a correlational design in this study, marital satisfaction cannot simply and certainly be affected by just predictor variables in this study. Another limitation of the present study was the research sample (married women of District 1 in Mashhad). It is suggested that empirical designs and larger samples be used in future research.

Findings of the present research, while expanding the research literature, highlight the necessity of considering physiological, cognitive and emotional characteristics in programs for the promotion of the relationships between couples and premarital counseling.

\section{Ethical Considerations}

\section{Compliance with ethical guidelines}

In order to observe ethical issues, after the participants' awareness of the goals and process of the research implementation, written consent was obtained from all participants and they were assured that the received information would remain completely confidential. This study has been conducted with the approval of the Research Deputy of Neyshabour Islamic Azad University.

\section{Funding}

This research did not receive any specific grant from funding agencies in the public, commercial, or not-forprofit sectors.

\section{Authors contributions}

The authors contributions is as follows: Conceptualization: Hossein Shareh, Maryam Eshaghi Sani; Research and investigation: Hossein Shareh, Maryam Eshaghi Sani; Edition and finalization of the text: Hossein Shareh; Resources: Hossein Shareh, Maryam Eshaghi Sani; and Project monitoring and management: Hossein Shareh.

\section{Conflict of interest}

The authors declared no conflict of interest.

\section{Acknowledgements}

Hereby, we offer our thanks to the respectable officials of Mashhad Municipality and the chief and staff of Rahyab Specialized Counseling Center for Mental Disorders and Sexual Dysfunctions in Mashhad, who assisted us in doing this research. 


\title{
نقش ييشبين شخصيت بامدادى رضامكاهى، انعطافيذيرى شناختى و تنظيم شناختى هيجان در رضايت زناشويي زنان ميانسال
}

\author{
• • \\ 1- دكتراي تخصصى، دانشيار، كروه علوم تربيتى، دانشكده ادبيات و علوم انسانى، دانشكاه حكيم سبزواري، سبزوار، ايران.

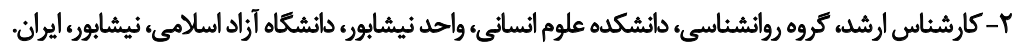

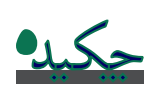

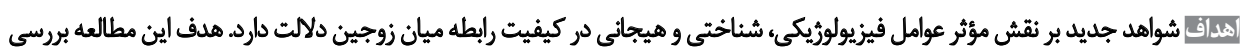

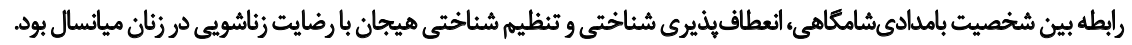

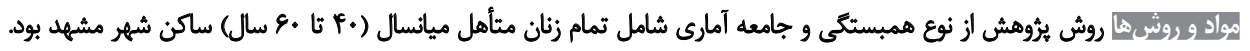

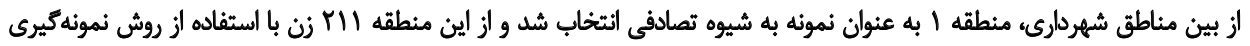

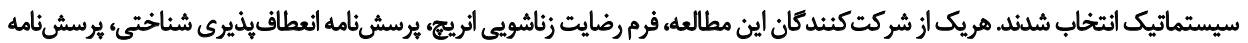

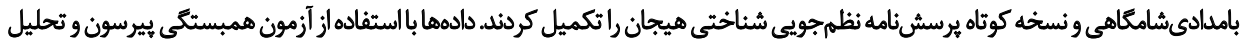
ركريسيون جندمتغيره تجزيه وتحليليل شيدند.

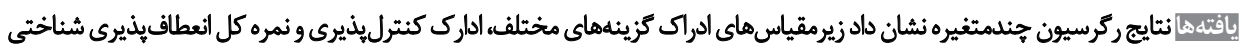

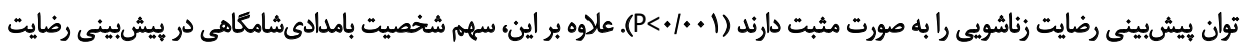

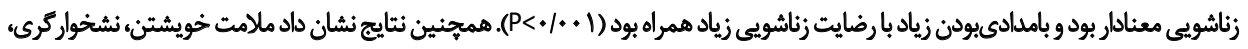

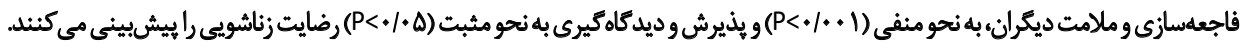

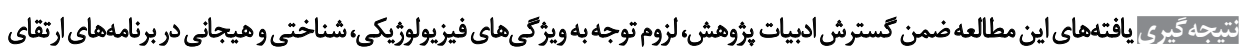

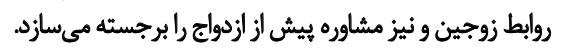

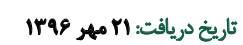
تاريخ يذيرش: 9 مرداد

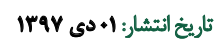

كليدوازهها: رضايت زناشويى،

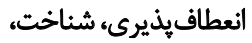
هيجان، بامدادى شامكاهئي

است. جرخههاى شبانهروزى' بر بسيارى از ابعاد فيزيولوريك،

مقدمه

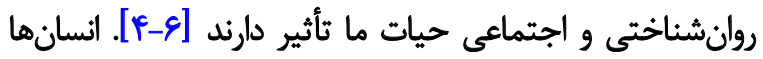

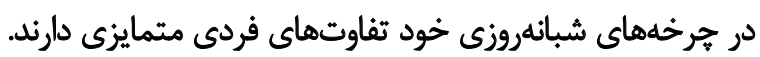

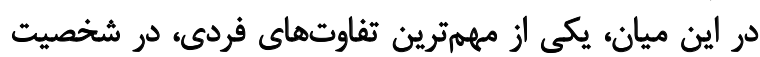

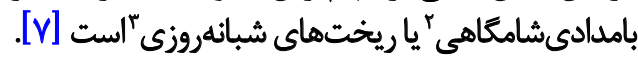

اقراد در طول بيوستارى از ترجيحات شبانهروزى قرار دارند كه

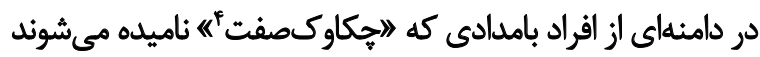

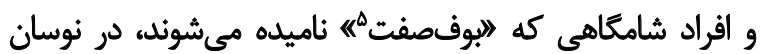

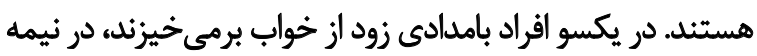

1. Circadian rhythm

2. Morningness-eveningness personality

3. Circadian Typology

4. Lark

5. Owl

$$
\text { … }
$$

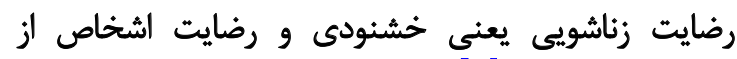

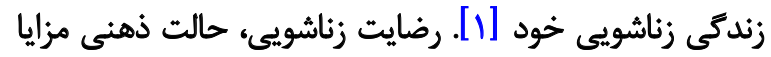

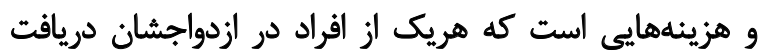

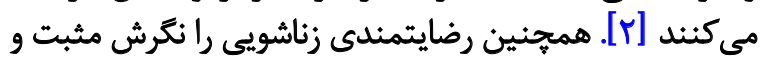

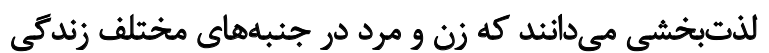
زناشويى تجربه مي كنيند [ب].

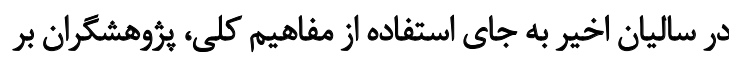

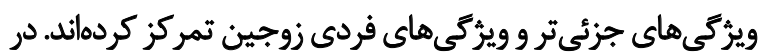

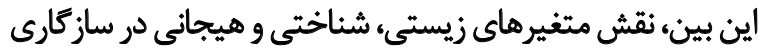

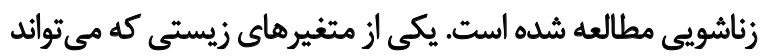

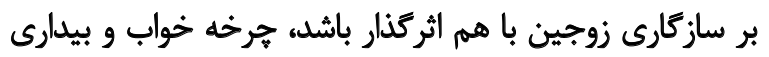

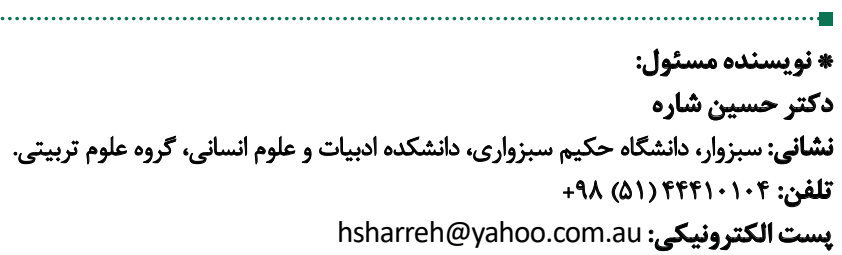




$$
\text { تابآورى بالاترى دارند [19]. }
$$

علاوه بر متغيرهاى فيزيولوزيكيى و شناختى، يكى از مهمترين

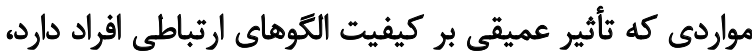

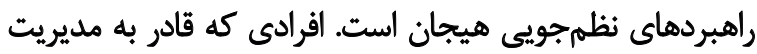

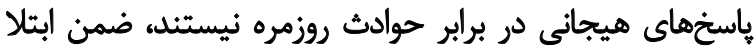

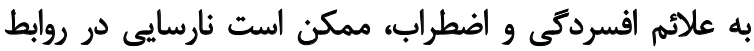

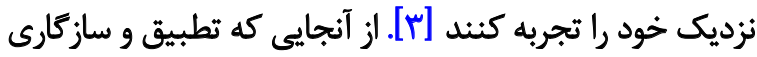

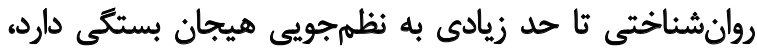

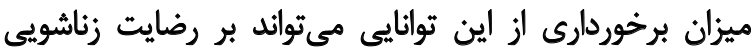
زوجين اثركذار باشد. با توجه به ارتباط بين انعطافيذيرى شناختى با سازكارى

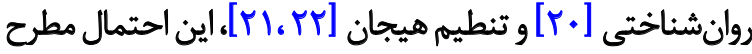

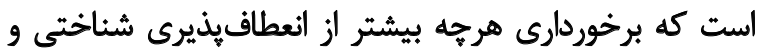

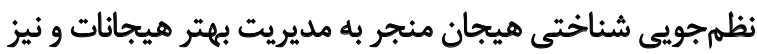

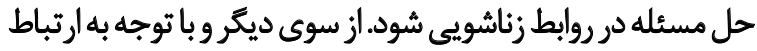
بين شخصيت بامدادى و برخوردارى از عملكرد شناختى و و هيجانى إنى

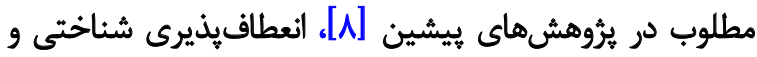
نظمجويى هيجان نيز ممكن است با شخصيت بامدادى بشامكاهيى رابطه داشته باشند.

شواهد نشان مىدهد رضايت زناشويى در ميانسالى نسبت به

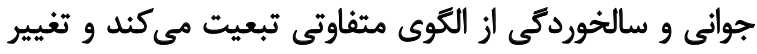

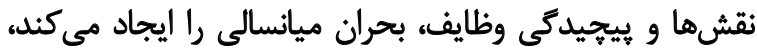

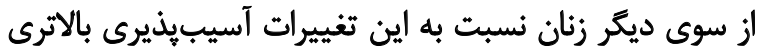

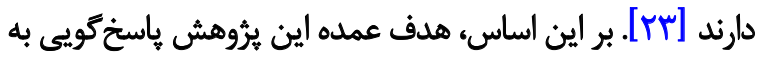

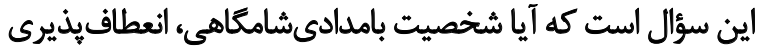

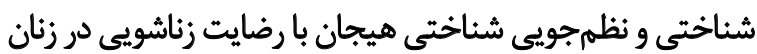

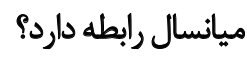

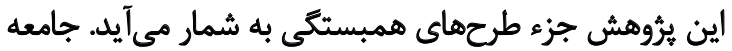

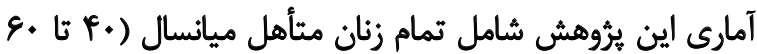

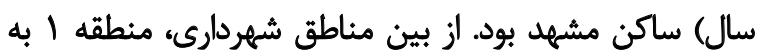

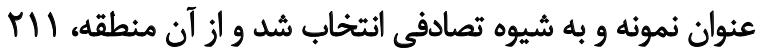

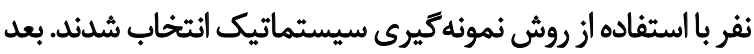

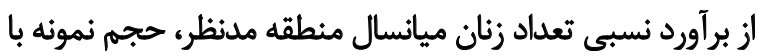

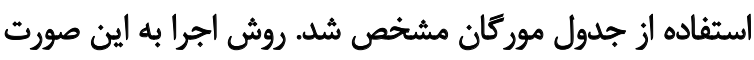

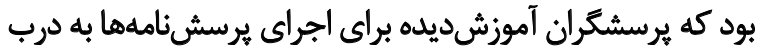

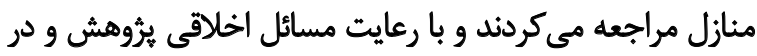

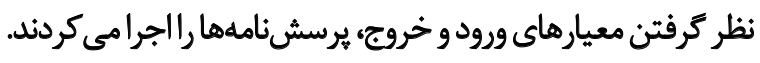

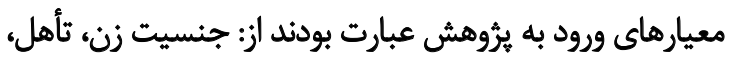

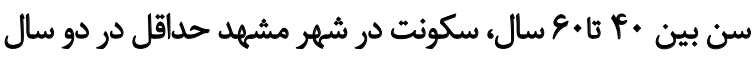

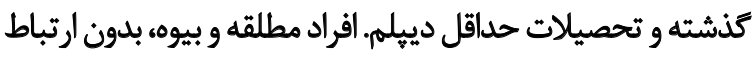

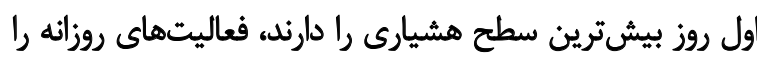
ترجيح مى دهند و دشوارى زيادى در خوابيدن ديروقت دارئد

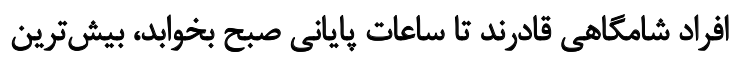

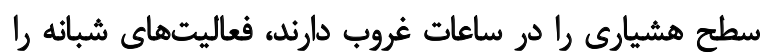

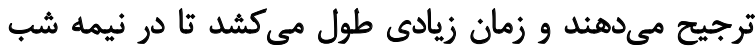

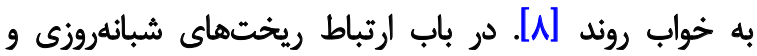

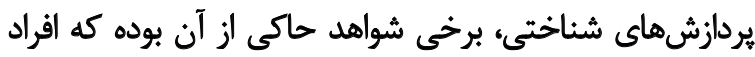

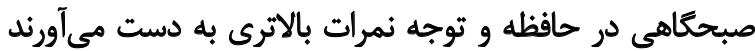

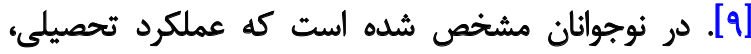

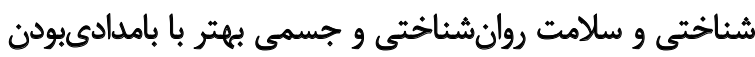
مرتبط است [·1]]

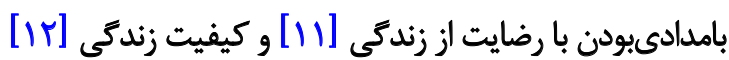

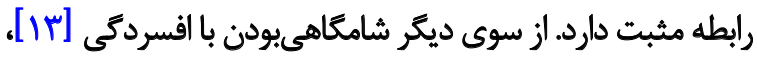

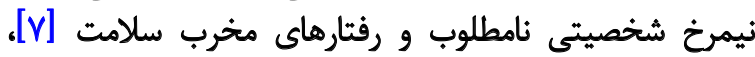

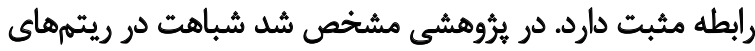

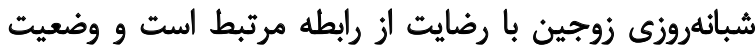

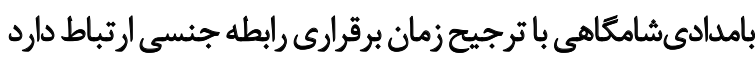

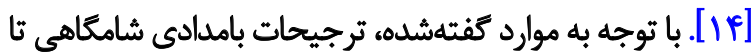

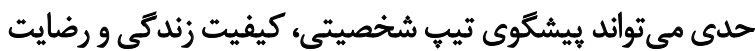

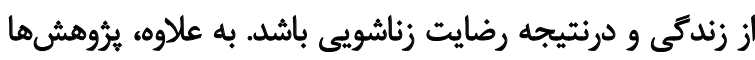

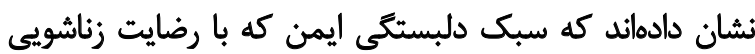

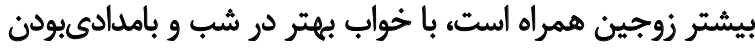

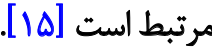

يكى ديكر از متغيرهايى كه ممكن است با ميزان رضايتمندى

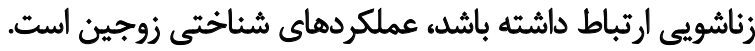

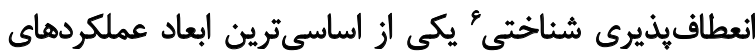

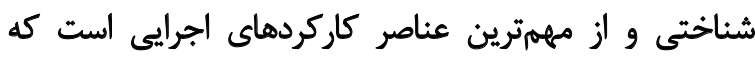

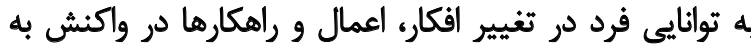

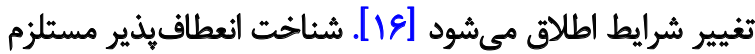

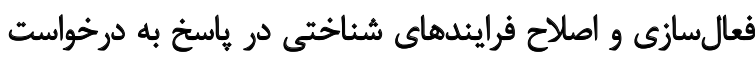

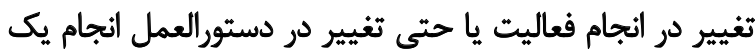

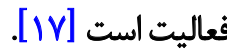

تونطافيذيرى شناختى به عنوان ويرُكى عمده شناخت انسان

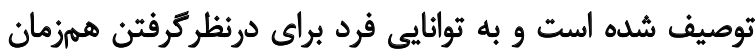

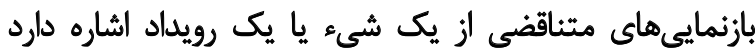

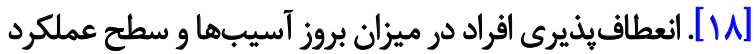

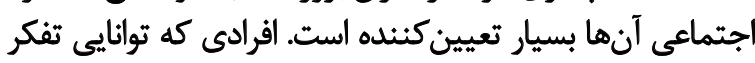

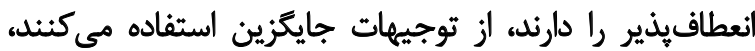

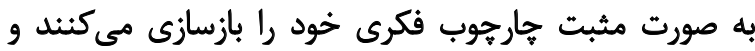

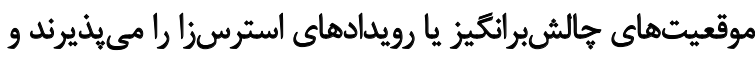

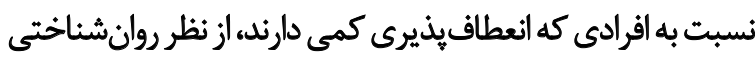

6. Cognitive flexibility 
توجيه جايكزّين براى رويدادهاى زندكى و رفتار انسانهاء توانايى

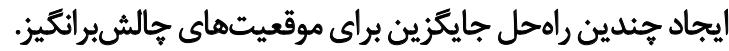
اين يرسشنامه در كار بالينى و غير بالينى و براي ارزيابى ميزان

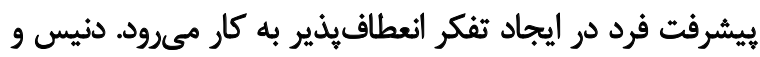

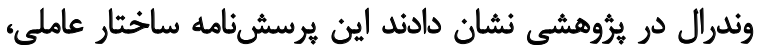

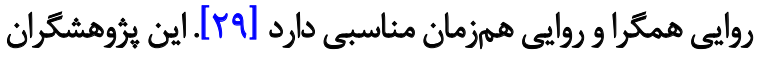

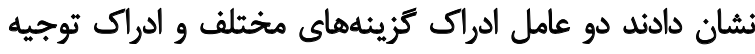

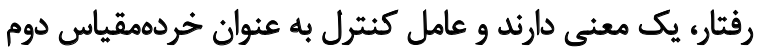

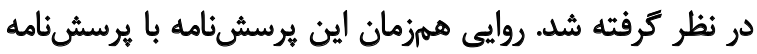

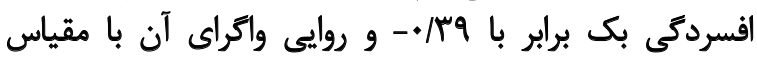

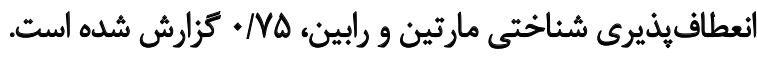

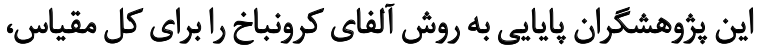

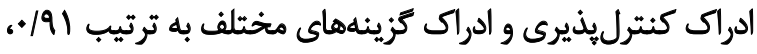

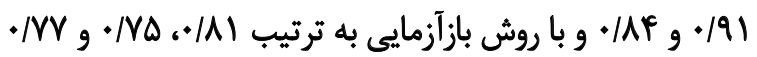
به دست آٓروند.

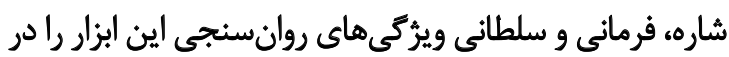

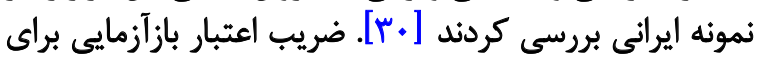

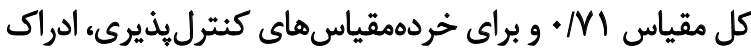

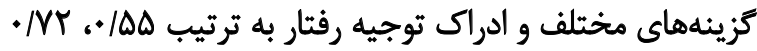

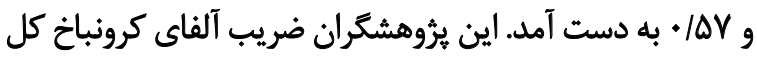

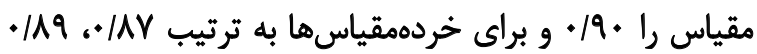

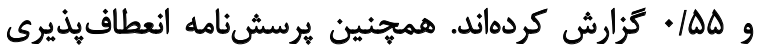

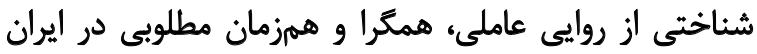

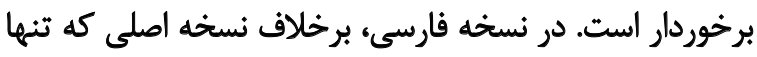

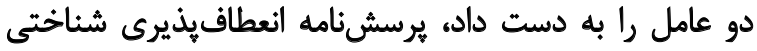

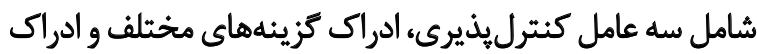

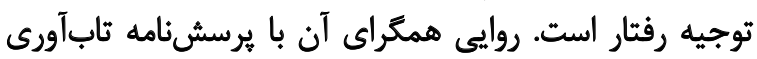

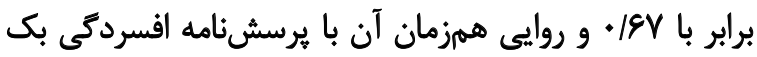

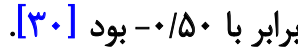

\section{يرسش بامهصبحكاهىشامكاهى}

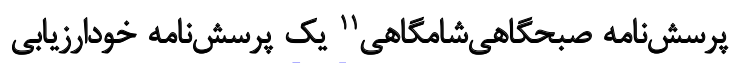

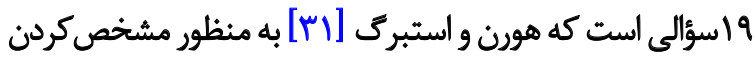

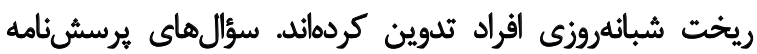
تزينهاي متفاوت ونمره

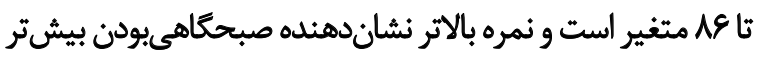

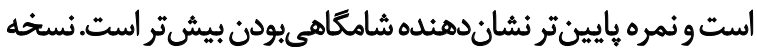

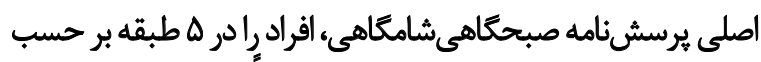

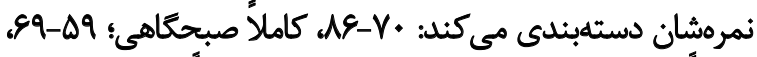

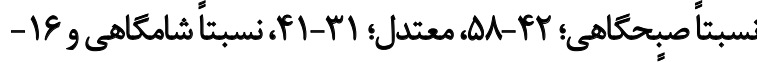

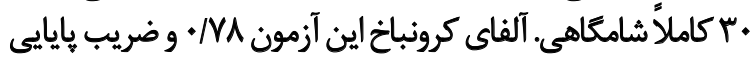

11. Morningness-Eveningness Questionnaire(MEQ)
جنسى با همسر، داراى روابط فرازناشويى، داراى افكار خودكشى داري

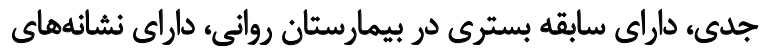

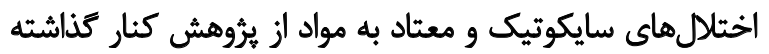

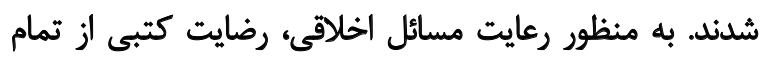

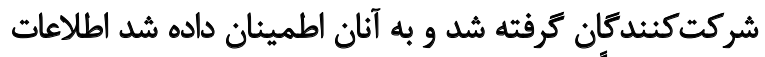

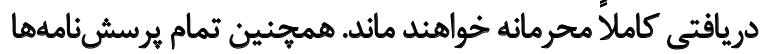
به صورت تصادفى و با نظم متغير به شركت كنيندكان ارائه شد.

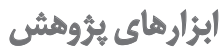

\section{فرم رضايت زناشويى انريج}

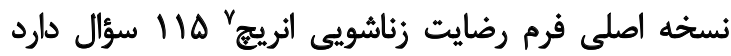

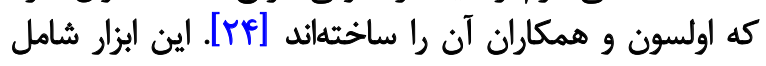

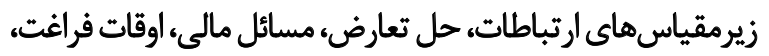

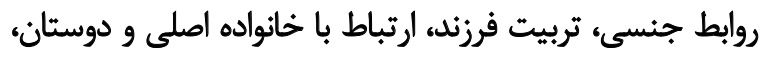

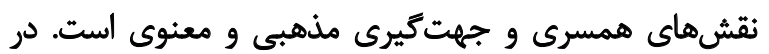

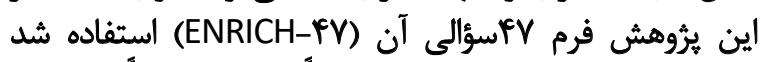

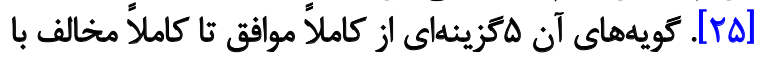

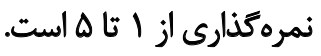

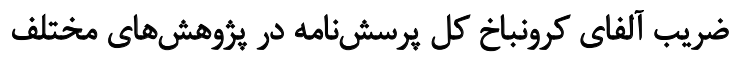

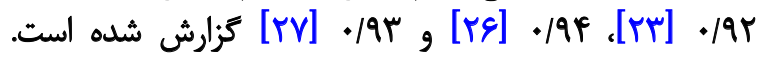

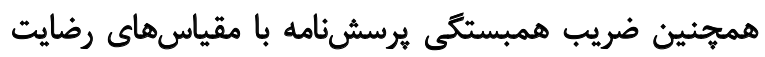

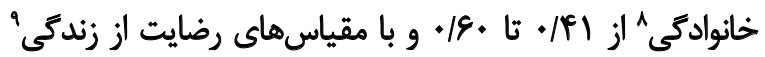

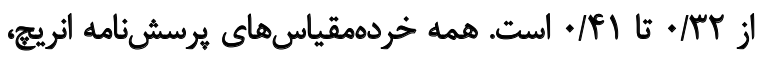

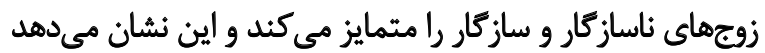

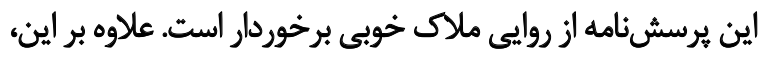

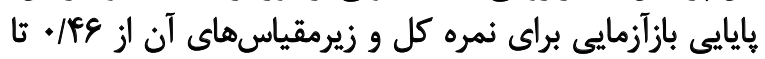

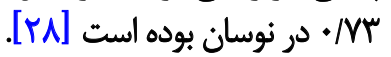

\section{هرسش نامه انعطاف بذيرى شناختي}

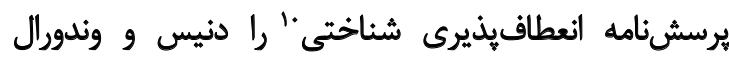

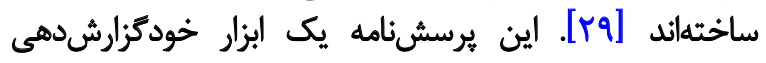

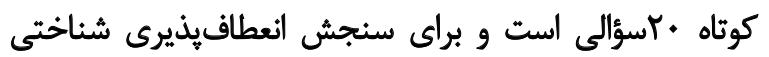

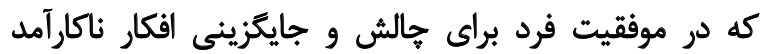

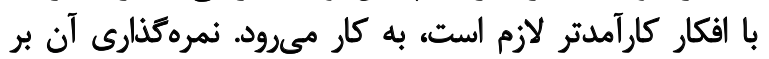

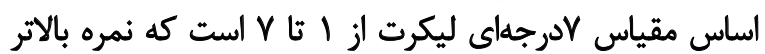

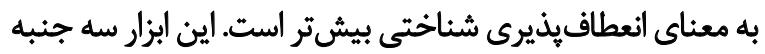

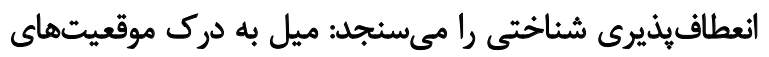

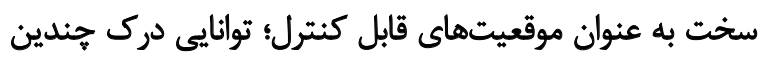

\footnotetext{
7. ENRICH Marital Satisfaction (EMS)

8. Family Satisfaction Scale

9. Life satisfaction

10. Cognitive Flexibility Scale(CFI)
} 


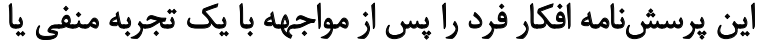

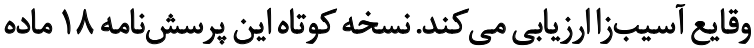

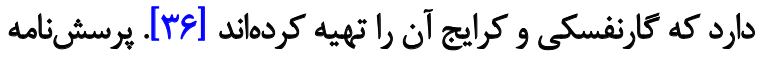

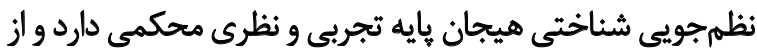
9 خردهمقياس تشكيل شده است

خردهمقياسهاى مذكور 9 راهبرد شناختى ملامت خويش،

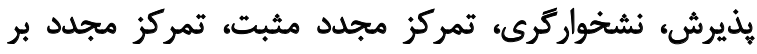

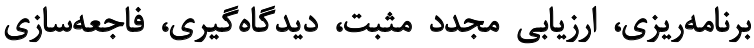

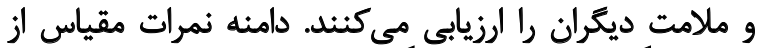

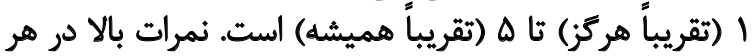

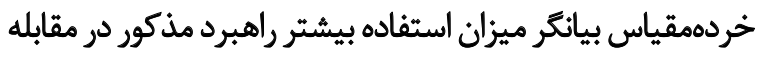

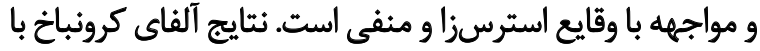

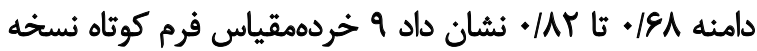

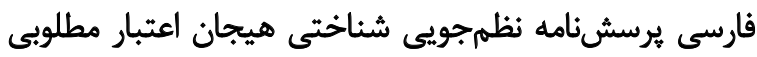
دارند.

تحليل مؤلفه اصلى ضمن تبيين VD درصد واريانس، از الكوى

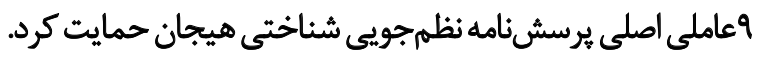

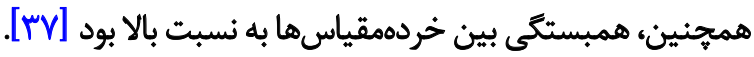

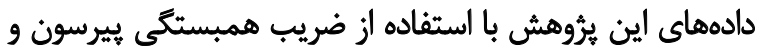

بازآزمايي آن MV • كزارش شده است [Tr]. در يُروهشى ديكر كاكي، دشاكس، آدان و ناتاله ميزان كلى إنى

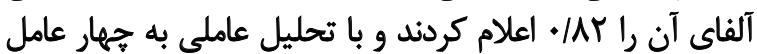

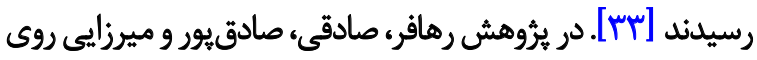

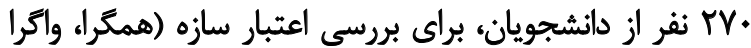

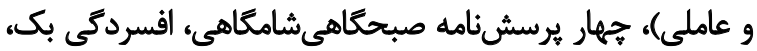

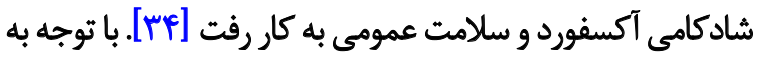

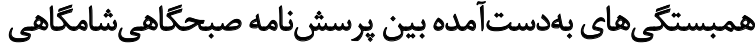

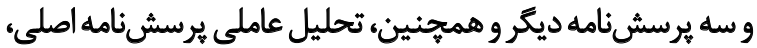

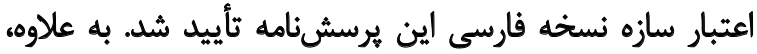

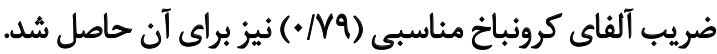

\section{نسخه كوتاه يرسش نامه نظمجويى شناختى هيجان}

يرسشنامه نظمجويى شناختى هيجان بان ا را كارنفسكى، كرايج

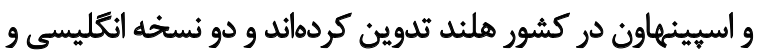

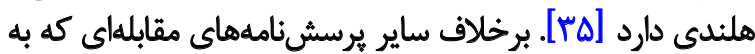
صورت آشكار بين افكار فرد واعمال واقعى او تمايز قائل نمى إشونده

12. Cognitive Emotion Regulation Questionnaire (CERQ)

جدول ا. ميائكين و انحراف استائدارد متغيرهاى يُروهش

\begin{tabular}{|c|c|c|}
\hline ميانكين土|نحراف معيار & مؤلفهها & مثغير -شاخص \\
\hline$|F P / A| \pm \mid \Delta / r V$ & & رضايت زناشويى \\
\hline SNVDIINAF & & بامدادى شامكاهى \\
\hline $1.197 \pm 8 / M^{2}$ & ملامت خويشتن & \multirow{9}{*}{ نتظيم شناختى هيجان } \\
\hline$N \cdot q \pm r / \Delta 8$ & ئيرش & \\
\hline $9 / 94 \pm \& / 9 q$ & نشخواركرى & \\
\hline$\Delta / \& N \pm 1 / r V$ & تمركز مجلد مثبت & \\
\hline$g / N \pm \pm / / V^{2}$ & تمركز بر برنامهريزى & \\
\hline$V / T V \pm Y / \Delta A$ & الرزيابى هجلد مثبت & \\
\hline$I r / \Delta \cdot \pm r / r \Delta$ & ديدكاهكيرى & \\
\hline $9 / V \Lambda \pm E / \& Y$ & فاجعdسازي & \\
\hline$V / A F \pm V / \cdot q$ & ملامت ديكران & \\
\hline$V / A V \pm T / T$. & ادراك توجيه رفتار & \multirow{4}{*}{ نعطافيذيرى ثناختى } \\
\hline$r \Delta / . q \pm \Delta / / f$ & ادراك كزيئههاى مختلف & \\
\hline$M \varphi / q . \pm 1 / \Lambda 1$ & ادراك كنترليذيرى & \\
\hline 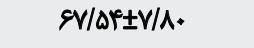 & تمره كل & \\
\hline
\end{tabular}


جدول r. ضرايب همبستكى بين رضايت زناشويى و انعطافيذيرى شئاختى

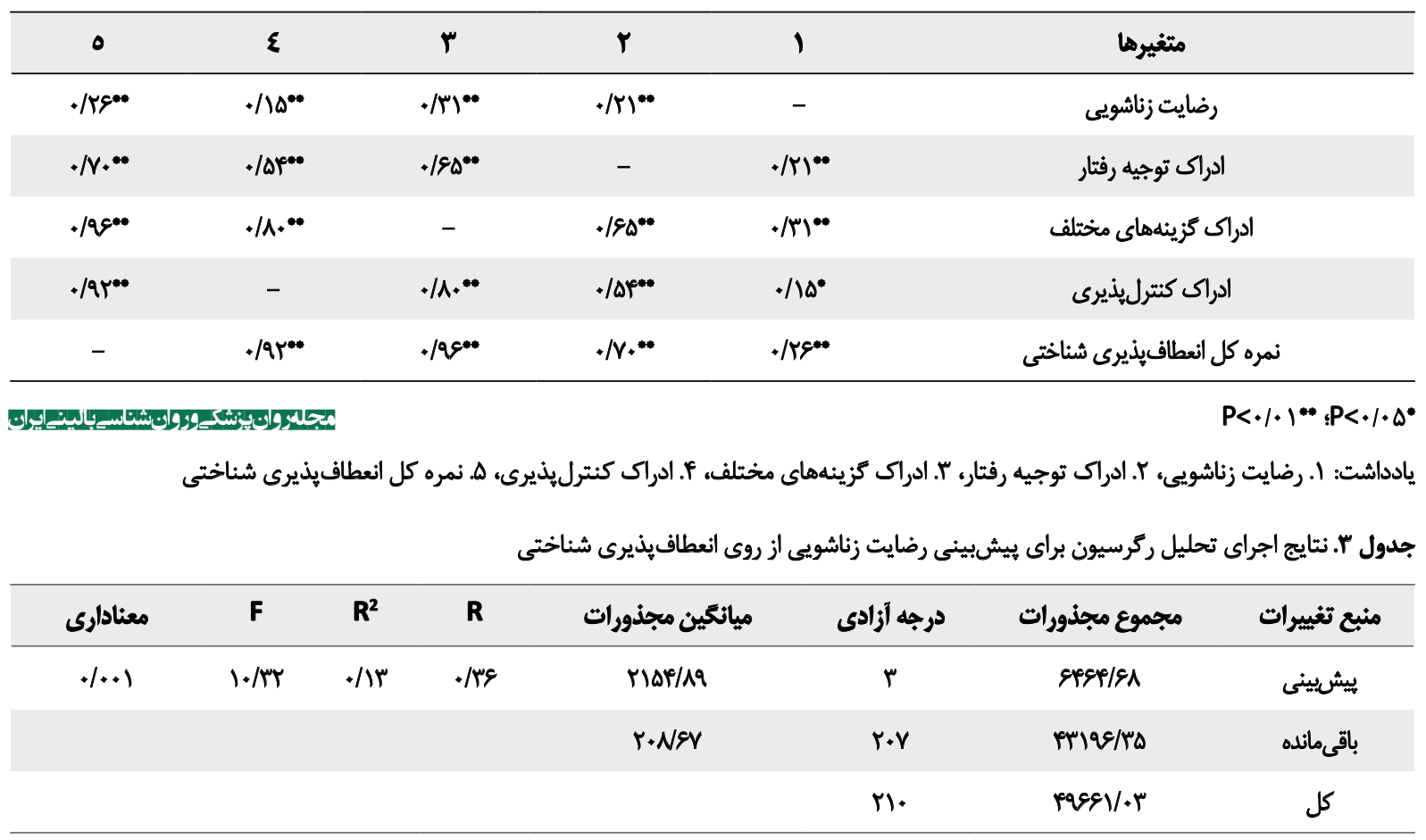

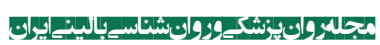

شناختى، در كام نخست ضرايب همبستگى بين دو متغير رضايت

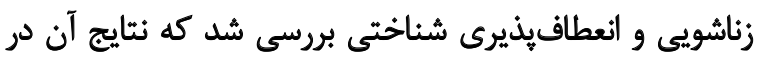

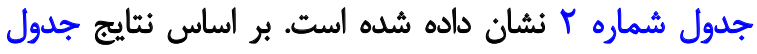

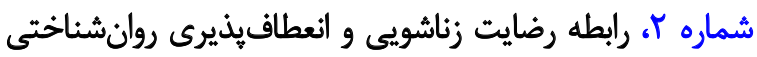

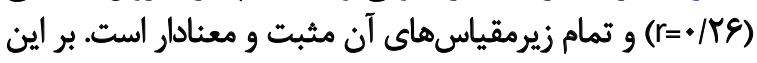

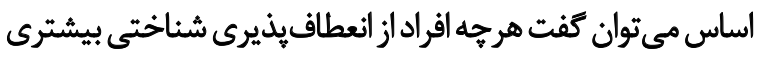

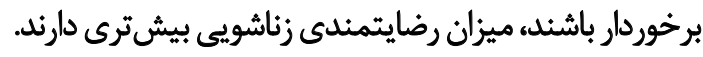
در كام بعدى به بررسى اثر يهيشبينى كنيندى انعطاف يذيرى

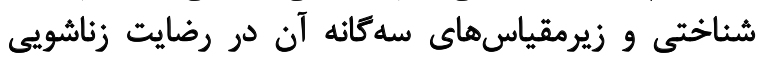

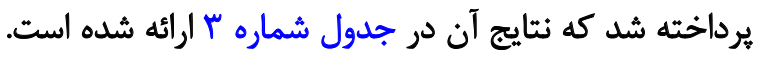

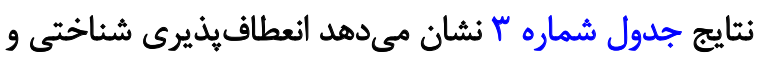

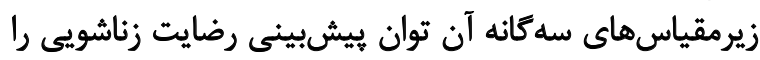

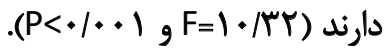

ركرسيون جندمتغيرى تجزيه و تحليل شد و از نرمافزار نسخه بr براى تحليل اطلاعات استفاده شد. تجني

Late

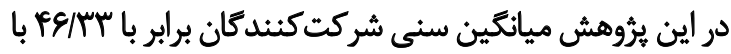

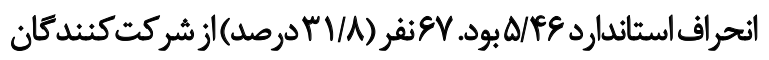

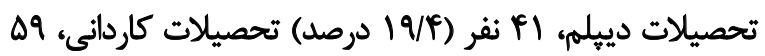

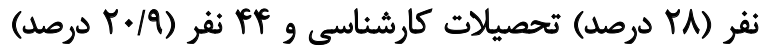

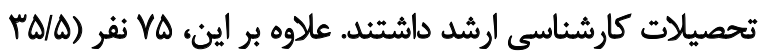

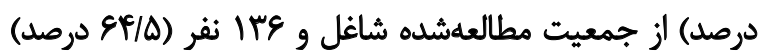

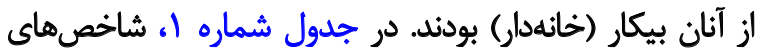
توصيفى مربوط به متغيرهاى يروهش ارائه شده است به منظور بررسى رابطه متغير رضايت زناشويى و انعطافٍ يذيرى

جدول F. ضرايب ركرسيون انعطافيذيرى شناختى در بيشبينى رضايت زناشويى

\begin{tabular}{|c|c|c|c|c|c|}
\hline Sig. & $\mathbf{t}$ & b & SE. & B & مثغيرهاى ييشبين \\
\hline$+1+1$ & r/99 & $+\Lambda$ & $+/ 4 \lambda$ &.$/ 199$ & ادراك توجيه رفتار \\
\hline $.1 .+1$ & $m / F i$ & $\cdot / n$ & $\cdot / \pi t$ & / & ادراى كزينههاى مختلف \\
\hline $.1 .+1$ & $r / \lambda$. & . & $+/ 10$ & . $/ \Delta r$ & ادراى كتترليذيرى \\
\hline $.1 . .1$ & e/pr &.$/ \% \Delta$ &.$/ M Y$ &.$/ \Delta \Delta$ & نمره كل انعطافيذيرى شناختمى \\
\hline
\end{tabular}

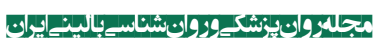


جدول ه. نتايج اجراي تحليل ركرسيون براي بيشبينى رضايت زناشويى از روى شخصيت بامدادىشامكاهى

\begin{tabular}{|c|c|c|c|c|c|c|c|}
\hline معنادارى & $\mathbf{F}$ & $\mathbf{R}^{2}$ & $\mathbf{R}$ & مياتكين مجذورات & درجها أزادى & مجموع مجذورات & منبع تغييرات \\
\hline \multirow[t]{3}{*}{$+1+.1$} & $|q / \mu|$ & $* / M$ & ./AT & $9 .+9 / r$ & 1 & $9+.9 / 4$ & ييشيينى \\
\hline & & & & $199 / \Delta$ & $r \cdot q$ & P.FAV/Rq & باقي مائده \\
\hline & & & & & M. & eqsey/.r & كل \\
\hline
\end{tabular}

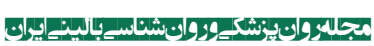

جدول ه. ضرايب ركرسيون شخصيت بامدادىشامكاهى در بيشيينى رضايت زناشويى

\begin{tabular}{|c|c|c|c|c|c|}
\hline Sig. & $\mathbf{t}$ & b & SE. & B & متغير هاي ييشيبن \\
\hline $.1 .+1$ & $8 / \Lambda$. & . $/ \mathrm{TH}$ & r/eq & $\cdot / \pi \Delta$ & شخُصيت بامدادي شامكاهي \\
\hline
\end{tabular}

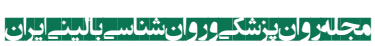

با توجه به معنادارى ملل فوق، براي بررسي دقيقتر و تعيين

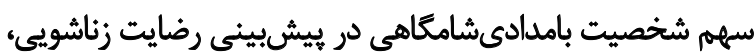

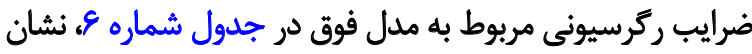

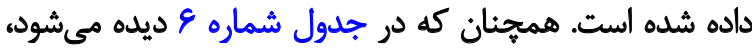

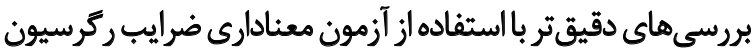

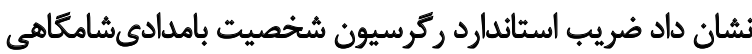

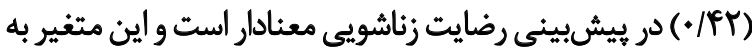

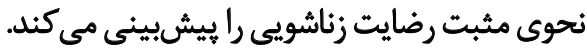

در كام بعدى، ضريب همبستكى بين دو متغير رضايت زناشويى و

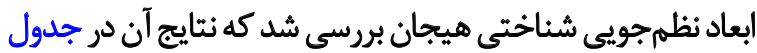

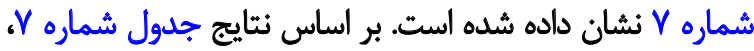

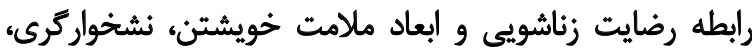

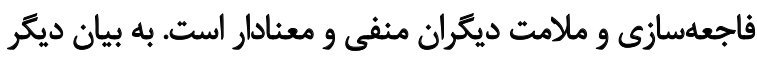

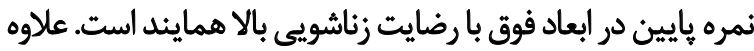

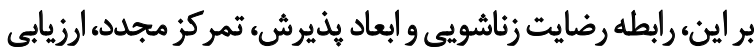

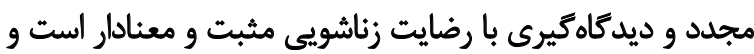

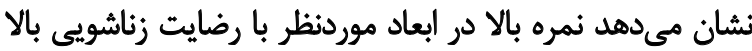

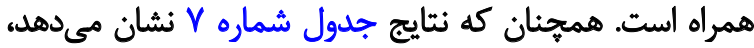

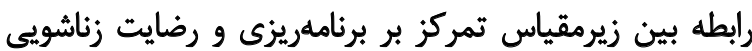
معنادار نيست.

در كام بعدى به بررسي اثر يهيشبينى كنيدكى زيرمقياسهاي

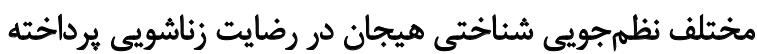

با توجه به معنادارى مدل فوقء براي بررسي دقيقتر و تعيين

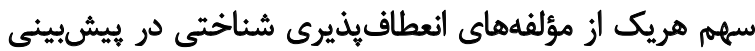

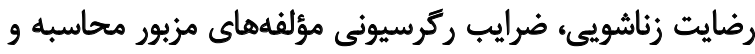

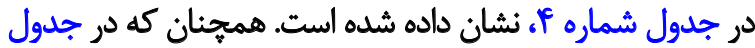

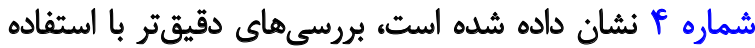

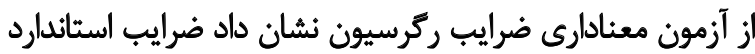

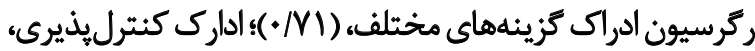

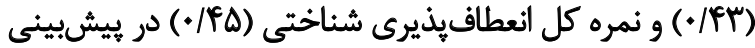

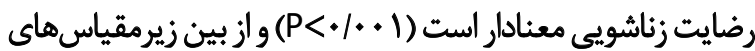

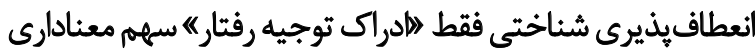
در يُيشبينى رضايتمندى زناشويى ندارد.

به منظور بررسى رابطه رضايت زناشويى و شخصيت

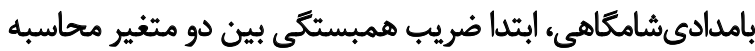

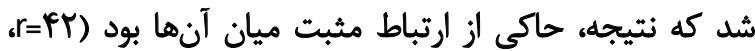

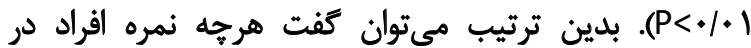

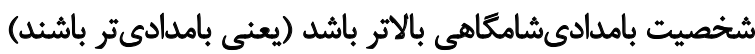

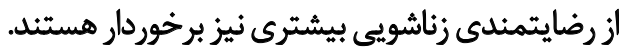

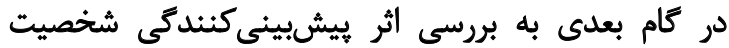

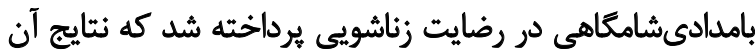

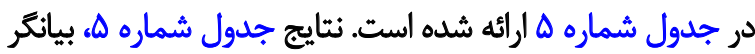

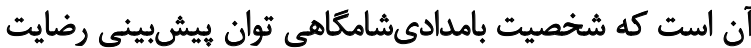
زناشويى را دارد (P)

جدول V. ضرايب همبستكى بين رضايت زناشويى و ابعاد نظمجويى شناختى هيجان

\begin{tabular}{|c|c|c|c|c|c|c|c|c|c|}
\hline ديمالامت & فاجعهازي & ديدكاهكيرى & ارزيديد & بونامهريزيز & تمجركز & نشخخوار & هذيرش & هويشتيتن & متغيرها \\
\hline$-+/ \pi \gamma^{* *}$ &.$- / r \omega^{* *}$ & $\cdot / M I^{* *}$ & . &.$/ 11$ &.$/ 18$ & $-+/ m \gamma^{* *}$ & . &.$- /$ re** & رضايت زناشويى \\
\hline
\end{tabular}


جدول ^. نتايج اجراي تحليل ركرسيون براى بيشبينى رضايت زناشويى از روى نظمجويى شناختى هيجان

\begin{tabular}{|c|c|c|c|c|c|c|c|}
\hline معنادارى & $\mathbf{F}$ & $\mathbf{R}^{2}$ & $\mathbf{R}$ & ميانكين مجذورات & دوجه آزادى & مجموع مجذورات & هنبع تغييرات \\
\hline \multirow[t]{3}{*}{$+1+\infty 1$} & $\mathrm{~V} / \mathrm{Mr}$ &.$/ \pi r$ & $+/ 19$ & $|m+4 / \Delta|$ & 9 & $|r+9| / 8$ & ييشيينى \\
\hline & & & & $M A / N$ & 198 & reALF & باقى مانده \\
\hline & & & & & $r .9$ & FANVA/E & كل \\
\hline
\end{tabular}

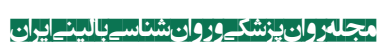

جدول 9. ضرايب ركرسيون نظمجويى شناختى هيجان در بيشبينى رضايت زناشويى

\begin{tabular}{|c|c|c|c|c|c|}
\hline Sig. & $\mathbf{t}$ & b & SE. & B & هتغيرهاي ييشيين \\
\hline $.1 .+1$ & $-t / R A$ &.$- / 18$ &.$/ K 8$ & $-\cdot / \Delta r$ & ملامت خويشتن \\
\hline $.1 . .1$ & $m / F i$ &.$/ 4$ &.$/ M I$ &.$/ 18$ & ليذيرش \\
\hline $.1 .+1$ & $-r / q)$ & $-+/ 17 q$ & ( ) & $-+1 \Delta V$ & نشخْواركرى \\
\hline$+1 * Y$ & $1 / M$ &.$/ 9$ & $\cdot / \pi$ &.$/ 18$ & تمركز مجلد مثبت \\
\hline$+M 11$ & $\cdot / \pi \wedge$ &.$/ 10$ & ./AS & +1 & تمركز بر برنامهريزى \\
\hline $.1 \cdot A$ & $M$ & $\cdot / V$ & . $/ \Delta T$ & .118 & الزيابي مجدد مثبت \\
\hline .1.$)^{2}$ & $r / \cdot A$ &.$/ 19$ &.$/ M A$ &.$/ 1 \% \Delta$ & ديدكاهكيرى \\
\hline $.1 . .1$ & -t/fA & $-.10 \Delta$ & .119 &.$- \mid 81$ & فاجعهسازى \\
\hline $.1 .+1$ & $-r / r q$ & $-\infty 10$ &.$/ T \Delta$ & $-\cdot / \Delta r$ & ملامت ديكران \\
\hline
\end{tabular}

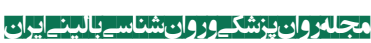

روانشئاختى و تمام زيرمقياسهاي آن مثبت و معنادار است.

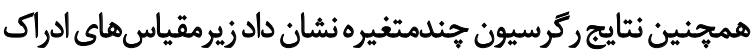

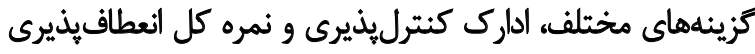

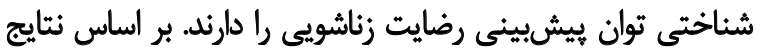

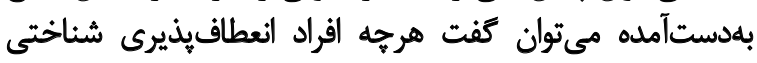
بيشترى داشته باشند، ميزان رضايثمندى زناشتونى دارند. تاكنون مطالعهاى به طور مستقيم رابطه رضايت زناشويني و انعطاف يذيرى شناختى را بررسى نكرده است. با اين حال، نتايج اين باين

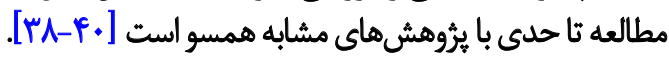
بنابر ديدكاه گيورت و همكاران انعطافيذيرى شناختى يكى از ابعاد كاركردهاي اجرايى است كه به توانايى تغيير اعمال و افكار مطابق

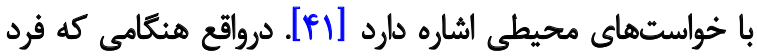
از راهبردهاى شناختى براى تغيير رفتار و انطباق با محيط استفاده الهاه

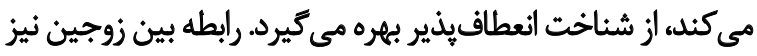

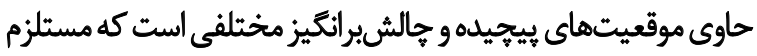

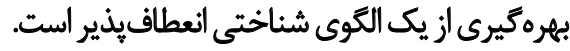

بر اساس نظريه تعارض [Fr]، وجود تعارض در ارتباطات

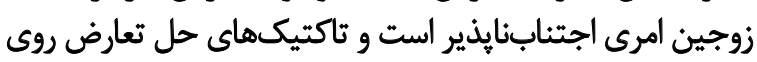

شد كه نتايج آن در جدول شماره A ارائه شده است. نتايج جدول

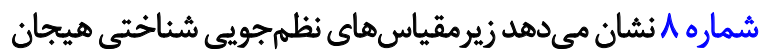
توان بيش بيني رضايت زناشويي را دارند (

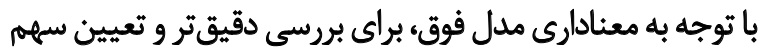

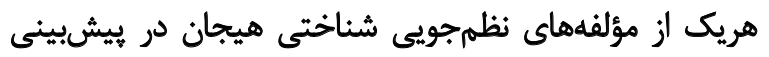

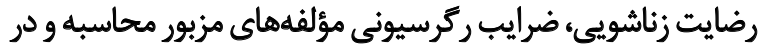

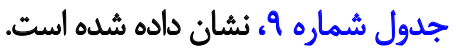

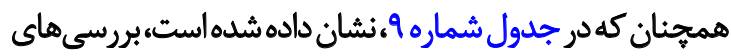

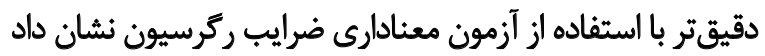

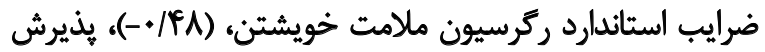

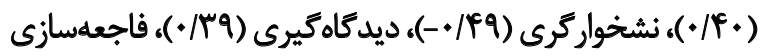

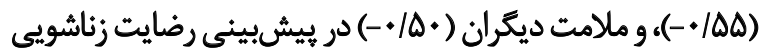

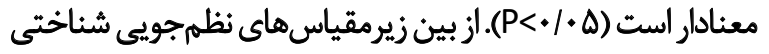

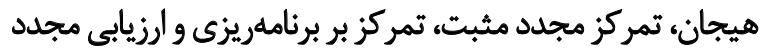

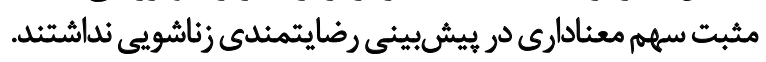
$\stackrel{\leftrightarrow}{\leftrightarrow}$

نتايج اين مطالعه نشان داد رابطه رضايت زناشويي و انعطافيذيرى 
همراه است [ـ[1]] بر اساس مطالعه نائل و همكاران اقراد تيب

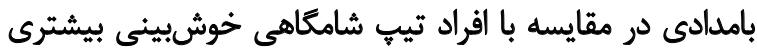

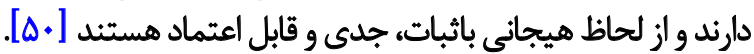
الكوى يافتهاى مذكور حكايت از آن دارد كه شخصيت

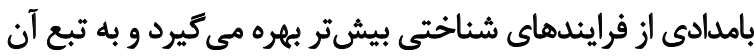

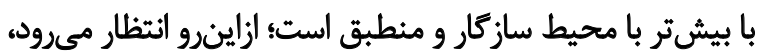

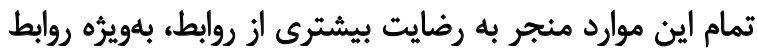

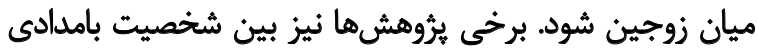

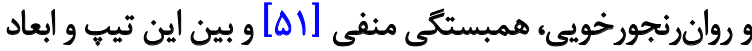

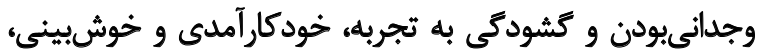

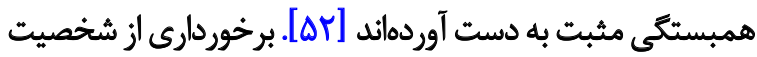

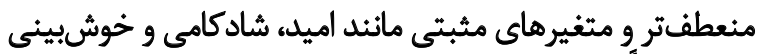
نيز احتمالأبا رضايت زناشويى رابطه مثبت دارديت مانديد

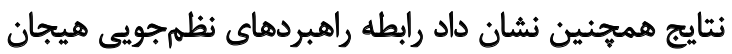

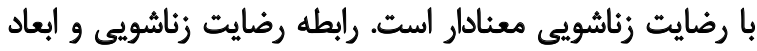

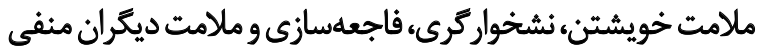

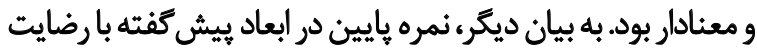

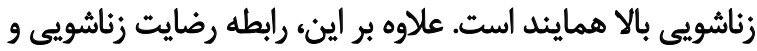

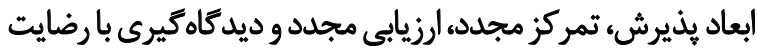

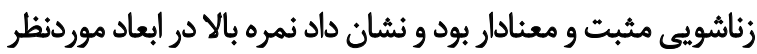
با رضايت زناشويى بالا همراه است.

نتايج بلهدستآمده در اين بخش با نتايج برخى از مطالعات

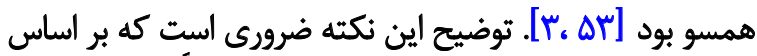

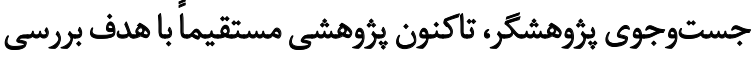

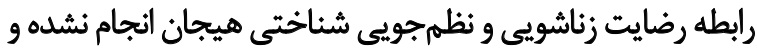

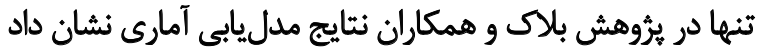

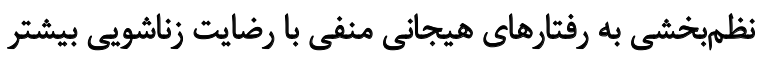

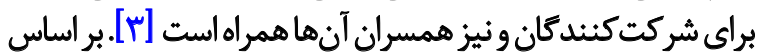

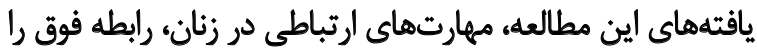

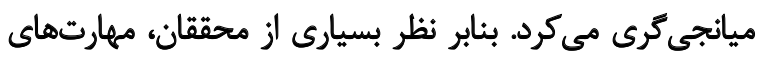

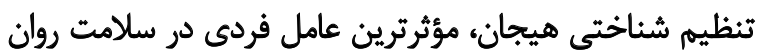

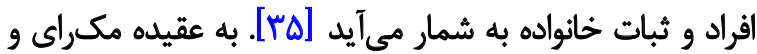

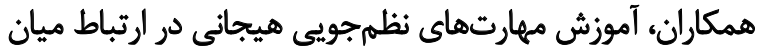

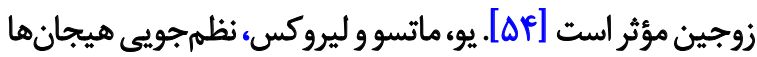
را مهمترين عامل وبيشبينى كنينده سازكارى مثبت (در زندئى

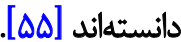

نظهجويى شناختى هيجان با هدف مديريت هيجان ها به منظور

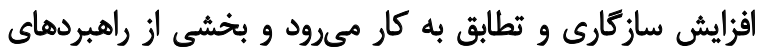

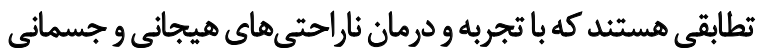

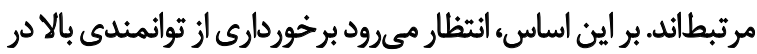

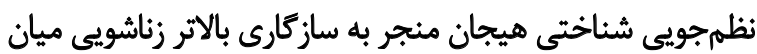

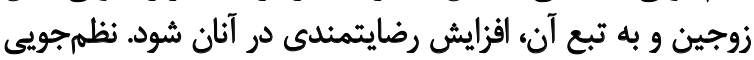

زيوستارى قرار دارد كه از تاكتيكهاى خشن (استفاده از اجبار و

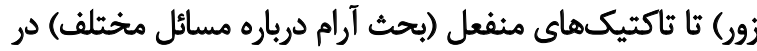

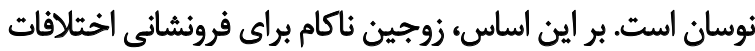

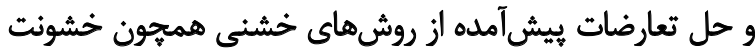

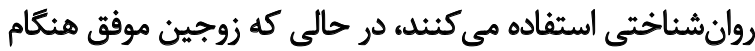

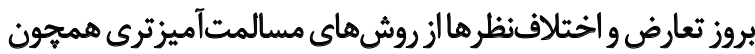

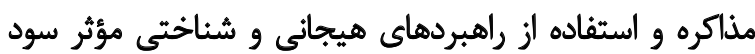

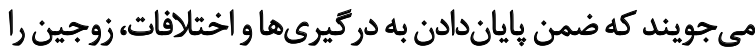

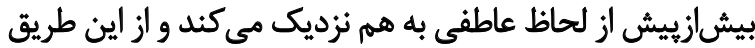

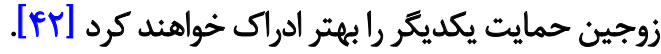

با توجه به رابطه ميان انعطافيذيرى شناختى و توانايى حل

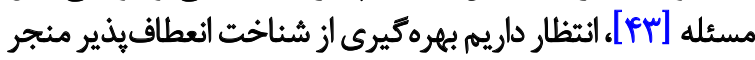

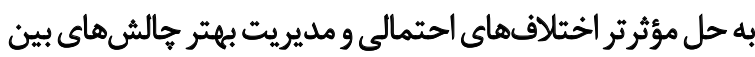

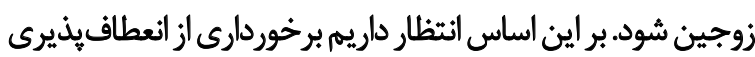
شناختى بالا با رضايتمندى زناشويى همراه باشد.

نتايج همجنين حاكى از ارتباطمثبت بين دومتغير رضايت زناشويى بإنيق

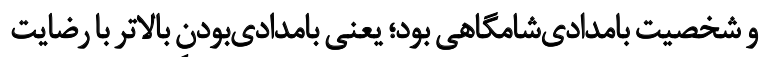

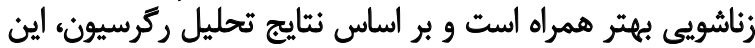

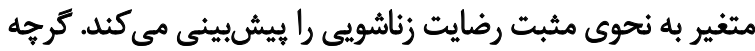

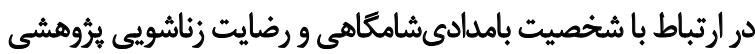

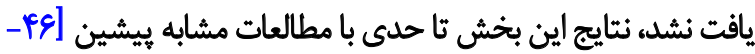

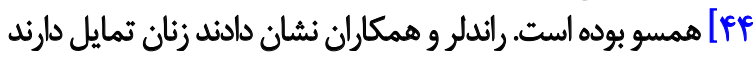

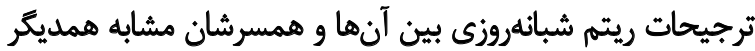

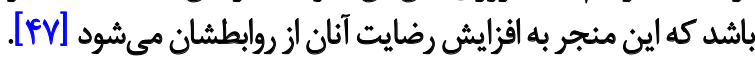

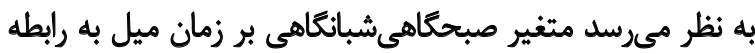
جنسى نيز تأثيركذار باشد [FN]

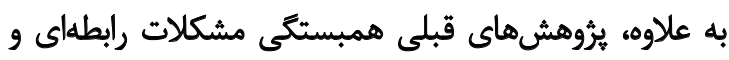

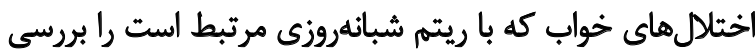

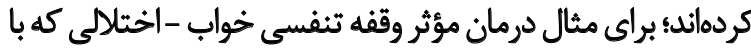

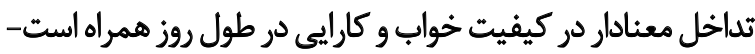

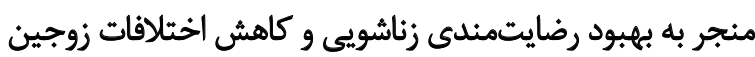

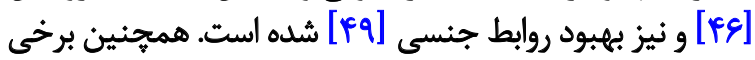

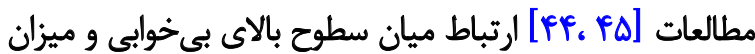

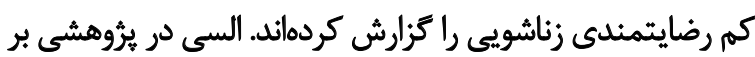

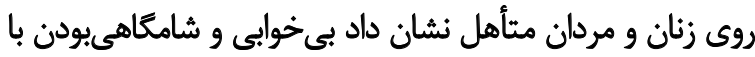

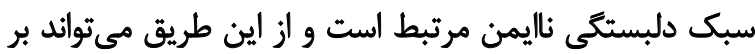

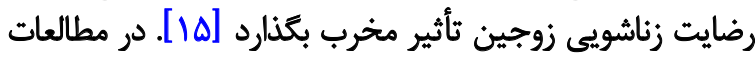

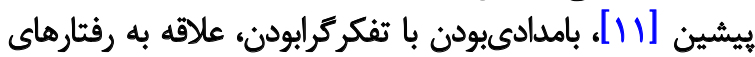

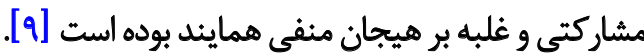

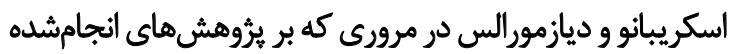

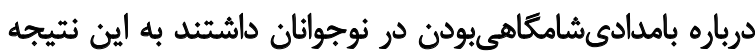

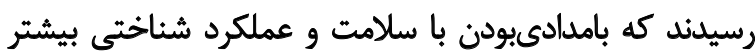


نمى توان بلهادكى و با قطعيت، رضايتمندى زناشويى را متأثر از متغيرهاى بيشبين در اين مطالعه دانست.

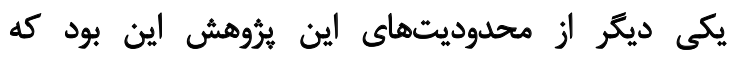

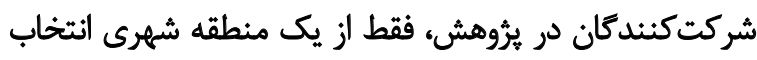

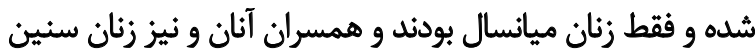

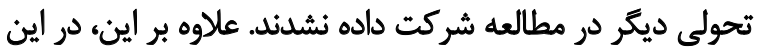

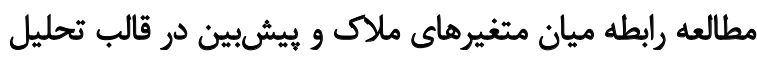

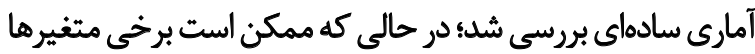

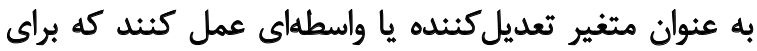

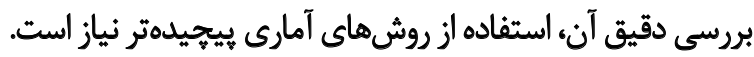

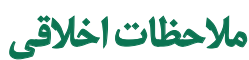

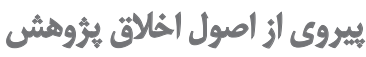

به منظور رعايت مسائل اخلاقى، يس از آكاهى شركت كنيندكان از

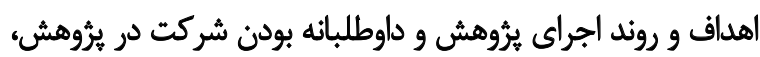

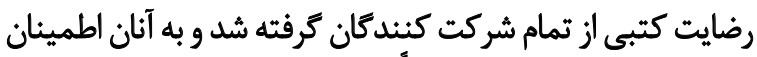
داده شد اطلاعات دريافتى كاملاً محرمانه خواهد ماند ماند.

$$
\text { حامي مالى }
$$

بنابر اظهار نويسنده مسئول، مقاله حامى مالى ندارد.

$$
\text { مشاركت نويسند مكان }
$$

مشاركت نويسندكان مقاله به اين شرح است: مفهومسازى:

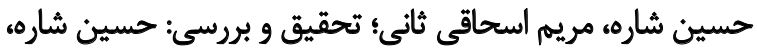

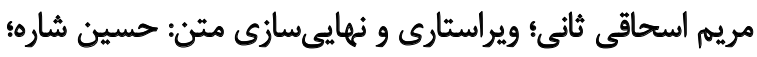

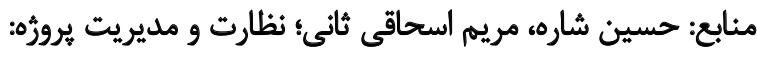

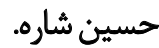
تعارض منافع

بنابر اظهار نويسنده مسئول، اين مقاله تعارض منافع ندارد و با بإنا

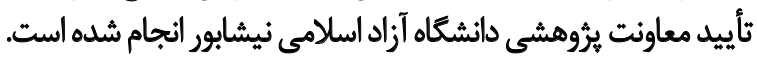

\section{سياسكَّرازى}

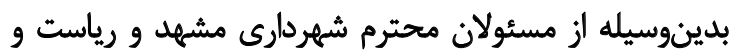

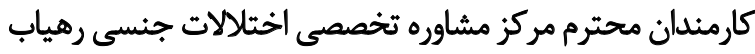
مشهد كه در اين يرؤش ماركز ما ا يارى كردند تشكر مي كثيه.

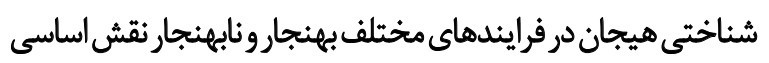

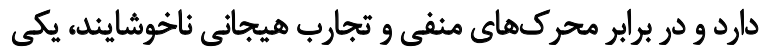

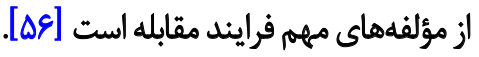

با توجه به ارتباط ميان مهارتهاي مقابلهاي و وضايت زناشوئي

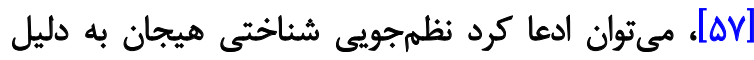

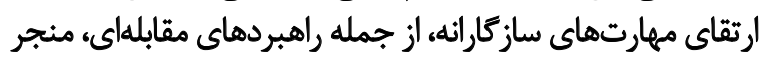

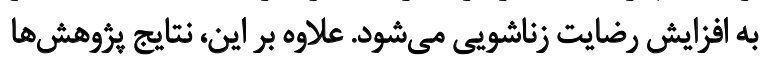

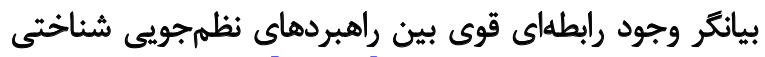

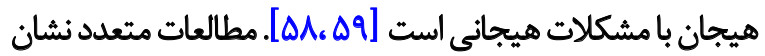

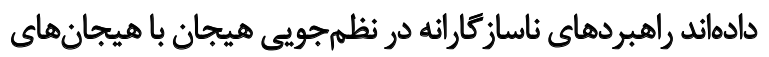

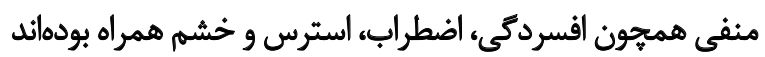

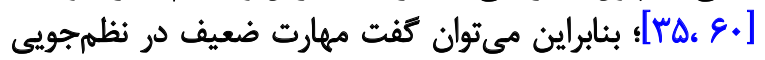

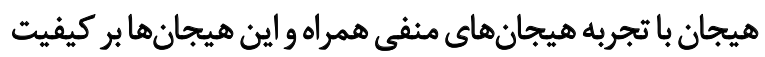

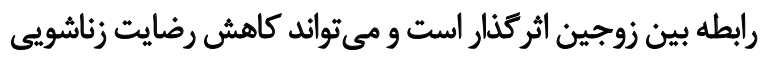

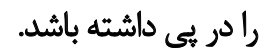

$$
\text { نتيجليَّيرى }
$$

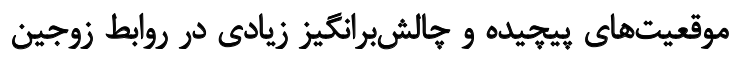

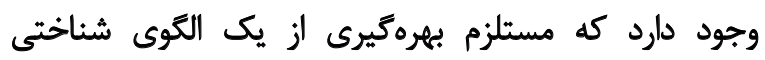

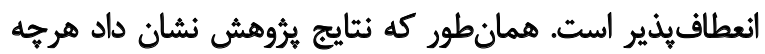

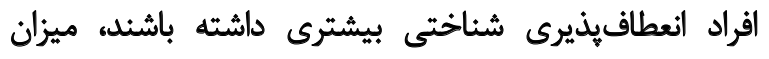

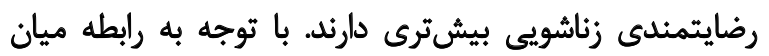

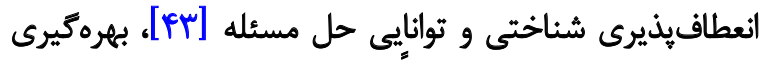

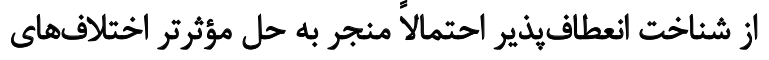

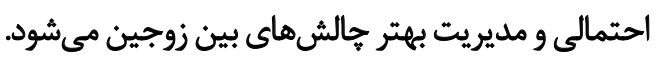

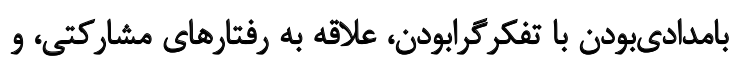

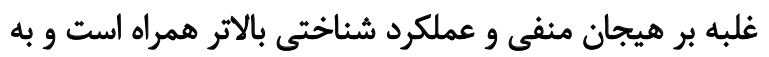

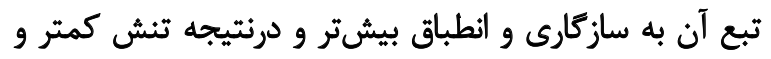

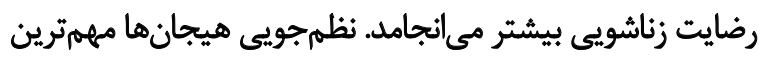

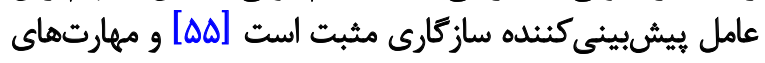

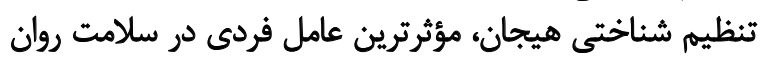

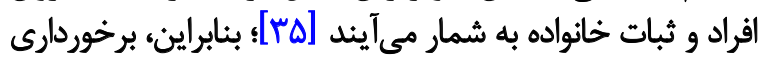

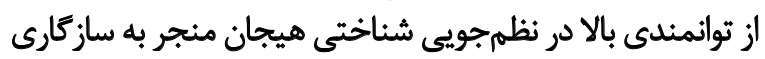

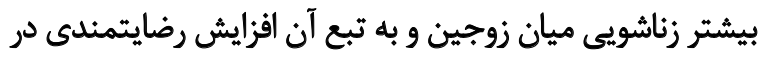
آنان مىشود.

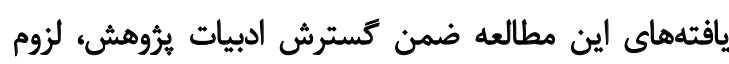

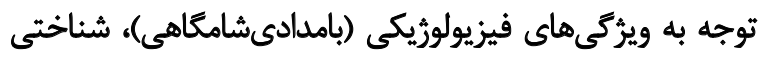

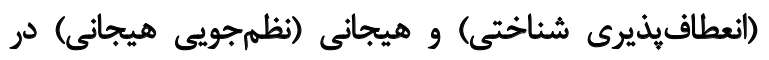

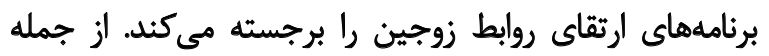

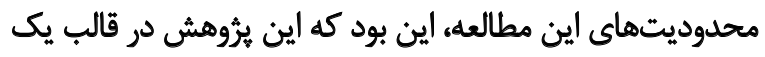

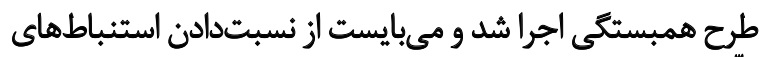

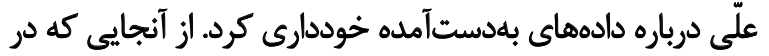
طرحهاى همبستكى همواره موضوع لاعليت دوسويه) مطرح است، 


\section{References}

[1] Bagwell EK. Factors influencing marital satisfaction with a specific focus on depression [MSc. thesis]. Ypsilanti, Michigan: Eastern Michigan University; 2006

[2] Lavner JA, Karney BR, Bradbury TN. Does couples' vommunication predict marital satisfaction, or does marital satisfaction predict communication. Journal of Marriage and Family. 2016; 78(3):680-94. [DOI:10.1111/jomf.12301] [PMID] [PMCID]

[3] Bloch L, Haase CM, Levenson RW. Emotion regulation predicts marital satisfaction: More than a wives' tale. Emotion. 2014; 14(1):130-44. [DOI:10.1037/a0034272] [PMID] [PMCID]

[4] Lange T, Dimitrov S, Born J. Effects of sleep and circadian rhythm on the human immune system. Annals of the New York Academy of Sciences. 2010; 1193(1):48-59. [DOI:10.1111/j.1749-6632.2009.05300.x] [PMID]

[5] Wulff K, Gatti S, Wettstein JG, Foster RG. Sleep and circadian rhythm disruption in psychiatric and neurodegenerative disease. Nature Reviews. Neuroscience. 2010; 11(8):589-99. [DOI:10.1038/nrn2868] [PMID]

[6] Van Veen MM, Kooij JS, Boonstra AM, Gordijn MC, Van Someren EJ. Delayed circadian rhythm in adults with attention-deficit/hyperactivity disorder and chronic sleeponset insomnia. Biological Psychiatry. 2010; 67(11):1091-6. [DOI:10.1016/j.biopsych.2009.12.032] [PMID]

[7] Urbán R, Magyaródi T, Rigó A. Morningness-eveningness, chronotypes and health-impairing behaviors in adolescents. Chronobiology International. 2011; 28(3):238-47. [DOI:10.3109/07420528.2010.549599] [PMID] [PMCID]

[8] Beşoluk Ş, Önder İ, Deveci İ. Morningness-eveningness preferences and academic achievement of university students. Chronobiology International. 2011; 28(2):118-25. [D OI:10.3109/07420528.2010.540729] [PMID]

[9] Cavallera GM, Giudici S. Morningness and eveningness personality: A survey in literature from 1995 up till 2006 Personality and Individual Differences. 2008; 44(1):3-21. [DOI:10.1016/j.paid.2007.07.009]

[10] Escribano C, Díaz Morales JF. Sleep habits and chronotype Effects on academic and cognitive performance in Spanish adolescents: A review. International Online Journal of Educational Sciences. 2016; 8(5):17-29. [DOI:10.15345/ iojes.2016.05.003]

[11] Díaz Morales JF, Jankowski KS, Vollmer C, Randler C. Morningness and life satisfaction: Further evidence from Spain. Chronobiology International. 2013; 30(10):1283-5. [D OI:10.3109/07420528.2013.840786] [PMID]

[12] Prieto PD, Díaz Morales JF, Barreno CE, Mateo MJ, Randler C. Morningness-eveningness and health-related quality of life among adolescents. The Spanish Journal of Psychology. 2012; 15(2):613-23. [DOI:10.5209/rev_ SJOP.2012.v15.n2.38872]

[13] Levandovski R, Dantas G, Fernandes LC, Caumo W, Torres I, Roenneberg T, et al. Depression scores associate with chronotype and social jetlag in a rural population. Chrono- biology International. 2011; 28(9):771-8. [DOI:10.3109/0742 0528.2011.602445] [PMID]

[14] Paulina J, Maciej S, Konrad SJ. Similarity in chronotype and preferred time for sex and its role in relationship quality and sexual satisfaction. Frontiers in Psychology. 2018; 9:443. [DOI:10.3389/fpsyg.2018.00443] [PMID] [PMCID]

[15] Elsey TL. The role of couple sleep concordance in subjective sleep quality: Attachment as a moderator of association [MSc. thesis]. Lexington, Kentucky: University of Kentucky; 2018.

[16] Whiting DL, Deane F, Ciarrochi J, McLeod H, Simpson G. Exploring the relationship between cognitive flexibility and psychological flexibility after acquired brain injury. Brain Injury. 2014; 28(5-6):646-7.

[17] Logue SF, Gould TJ. The neural and genetic basis of executive function: attention, cognitive flexibility, and response inhibition. Pharmacology Biochemistry and Behavior. 2014; 123:45-54. [DOI:10.1016/j.pbb.2013.08.007] [PMID] [PMCID]

[18] Happel MF, Niekisch H, Rivera LL, Ohl FW, Deliano $M$, Frischknecht R. Enhanced cognitive flexibility in reversal learning induced by removal of the extracellular matrix in auditory cortex. Proceedings of the National Academy of Sciences. 2014; 111(7):2800-5. [DOI:10.1073/ pnas.1310272111] [PMID] [PMCID]

[19] Gan Y, Zhang Y, Wang X, Wang S, Shen X. The coping flexibility of neurasthenia and depressive patients. Personality and Individual Differences. 2006; 40(5):859-71. [DOI:10.1016/j.paid.2005.09.006]

[20] Noal S, Levy C, Hardouin A, Rieux C, Heutte N, Ségura $\mathrm{C}$, et al. One-year longitudinal study of fatigue, cognitive functions, and quality of life after adjuvant radiotherapy for breast cancer. International Journal of Radiation Oncology Biology, Physics. 2011; 81(3):795-803.

[21] Kok BE, Fredrickson BL. Upward spirals of the heart: Autonomic flexibility, as indexed by vagal tone, reciprocally and prospectively predicts positive emotions and socia connectedness. Biological Psychology. 2010; 85(3):432-6. [DOI:10.1016/j.biopsycho.2010.09.005] [PMID] [PMCID]

[22] Velotti P, Balzarotti S, Tagliabue S, English T, Zavattini GC, Gross JJ. Emotional suppression in early marriage: Actor, partner, and similarity effects on marital quality. Journal of Social and Personal Relationships. 2016; 33(3):277302. [DOI:10.1177/0265407515574466]

[23] Nema S. Effect of marital adjustment in middle-aged adults. International Journal of Scientific and Research Publications. 2013; 3(9):1-6.

[24] Fowers BJ, Olson DH. ENRICH marital satisfaction scale: A brief research and clinical tool. Journal of Family Psychology. 1993; 7(2):176-85. [DOI:10.1037/0893-3200.7.2.176]

[25] Olson DH. Family Satisfaction Scale (FSS). Minneapolis, Minnesota: Life Innovations; 2004.

[26] Mahdavian F. [Fffect of education and relationship on marital satisfaction (Persian)] [MA. thesis]. Tehran: Tehran University of Medical Science; 1996.

[27] Soleimanian A. [Effect of irrational beliefs of marital satisfaction (Persian)] [MA. thesis]. Tehran: Kharazmi University; 1993. 
[28] Ameri, F. [Effect of strategical family therapy on marital conflicts (Persian)] [PhD. dessertation]. Tehran: Tarbiat Modares University; 2002.

[29] Dennis JP, Vander Wal JS. The cognitive flexibility inventory: Instrument development and estimates of reliability and validity. Cognitive Therapy and Research. 2010; 34(3):241-53. [DOI:10.1007/s10608-009-9276-4]

[30] Shareh H, Farmani A, Soltani E. Investigating the reliability and validity of the Cognitive Flexibility Inventory (CFII) among Iranian university students. Practice in Clinical Psychology. 2014; 2(1):43-50.

[31] Horne JA, Ostberg O. A self-assessment questionnaire to determine morningness-eveningness in human circadian rhythms. International Journal of Chronobiology. 1976; 4(2):97-110. [PMID]

[32] Taillard J, Philip P, Chastang JF, Bioulac B. Validation of horne and ostberg morningness-eveningness questionnaire in a middle-aged population of French workers. Journal of Biological Rhythms. 2004; 19(1):76-86. [DOI:10.1177/0748730403259849] [PMID]

[33] Caci H, Deschaux O, Adan A, Natale V. Comparing three morningness scales: Age and gender effects, structure and cut-off criteria. Sleep Medicine. 2009; 10(2):240-5. [DOI:10.1016/j.sleep.2008.01.007] [PMID]

[34] Rahafar A, Sadeghi Jujilee M, Sadeghpour A, Mirzaei S. Surveying psychometric features of Persian version of morning-eventide questionnaire. Clinical Psychology \& Personality. 2013; 2(8):109-22

[35] Garnefski N, Kraaij V, Spinhoven P. Negative life events, cognitive emotion regulation and emotional problems. Personality and Individual Differences. 2001; 30(8):1311-27. [DOI:10.1016/S0191-8869(00)00113-6]

[36] Garnefski N, Kraaij V. Cognitive Emotion Regulation Questionnaire-development of a short 18- item version (CERQ-short). Personality and Individual Differences. 2006; 41(6):1045-53. [DOI:10.1016/j.paid.2006.04.010]

[37] Hasani J. [The reliability and validity of the short form of the cognitive emotion regulation questionnaire (Persian)]. Journal of Research in Behavioural Sciences. 2011; 9(4):229-40.

[38] Lee JK, Orsillo SM. Investigating cognitive flexibility as a potential mechanism of mindfulness in generalized anxiety disorder. Journal of Behavior Therapy and Experimental Psychiatry. 2014; 45(1):208-16. [DOI:10.1016/j. jbtep.2013.10.008] [PMID]

[39] Dietrich A, Kanso R. A review of EEG, ERP, and neuroimaging studies of creativity and insight. Psychological Bulletin. 2010; 136(5):822. [DOI:10.1037/a0019749] [PMID]

[40] Rittle Johnson B, Star JR. Compared with what? The effects of different comparisons on conceptual knowledge and procedural flexibility for equation solving. Journal of Educational Psychology. 2009; 101(3):529-44. [DOI:10.1037/ a0014224]

[41] Geurts HM, Corbett B, Solomon M. The paradox of cognitive flexibility in autism. Trends in Cognitive Sciences. 2009; 13(2):74-82. [DOI:10.1016/j.tics.2008.11.006] [PMID] [PMCID]
[42] Straus MA. Blaming the messenger for the bad news about partner violence by women: The methodological, theoretical, and value basis of the purported invalidity of the Conflict Tactics Scales. Behavioral Sciences \& the Law. 2012; 30(5):538-56. [DOI:10.1002/bsl.2023] [PMID]

[43] Canas J, Quesada J, Antolí A, Fajardo I. Cognitive flexibility and adaptability to environmental changes in dynamic complex problem-solving tasks. Ergonomics. 2003; 46(5):482-501. [DOI:10.1080/0014013031000061640] [PMID]

[44] Tzischinsky O, Shochat T. Eveningness, sleep patterns, daytime functioning, and quality of life in Israeli adolescents. Chronobiology International. 2011; 28(4):338-43. [DO I:10.3109/07420528.2011.560698] [PMID]

[45] Strawbridge WJ, Shema SJ, Roberts RE. Impact of spouses' sleep problems on partners. Sleep. 2004; 27(3):527-31. [DOI:10.1093/sleep/27.3.527] [PMID]

[46] McFadyen TA, Espie CA, McArdle N, Douglas NJ, Engleman HM. Controlled, prospective trial of psychosocial function before and after continuous positive airway pressure therapy. European Respiratory Journal. 2001;18(6):9961002. [DOI:10.1183/09031936.01.00209301] [PMID]

[47] Randler C, Barrenstein S, Vollmer C, Díaz Morales JF, Jankowski KS. Women would like their partners to be more synchronized with them in their sleep-wake rhythm. The Spanish Journal of Psychology. 2014; 17:E70. [DOI: 10.1017/ sjp.2014.72] [PMID]

[48] Jankowski KS, Díaz Morales JF, Randler C. Chronotype, gender, and time for sex. Chronobiology International. 2014; 31(8):911-6. [DOI:10.3109/07420528.2014.925470] [PMID]

[49] Beninati W, Sanders MH. Optimal continuous positive airway pressure for the treatment of obstructive sleep apnea/hypopnea. Sleep Medicine Reviews. 2001; 5(1):7-23. [DOI:10.1053/smrv.2000.0131] [PMID]

[50] Natale V, Esposito MJ, Martoni M, Fabbri M. Validity of the reduced version of the morningness eveningness questionnaire. Sleep and Biological Rhythms. 2006; 4(1):72-4. [DOI:10.1111/j.1479-8425.2006.00192.x]

[51] Tsaousis I. Circadian preferences and personality traits: A meta-analysis. European Journal of Personality. 2010; 24(4):356-73. [DOI:10.1002/per.754]

[52] Randler C. Morningness-eveningness, sleep-wake variables and big five personality factors. Personality and Individual Differences. 2008; 45(2):191-6. [DOI:10.1016/j. paid.2008.03.007]

[53] Dalgleish TL, Johnson SM, Burgess Moser M, Lafontaine MF, Wiebe SA, Tasca GA. Predicting change in marital satisfaction throughout emotionally focused couple therapy. Journal of Marital and Family Therapy. 2015; 41(3):276-91. [DOI:10.1111/jmft.12101] [PMID]

[54] McRae TR, Dalgleish TL, Johnson SM, Burgess Moser M, Killian KD. Emotion regulation and key change events in emotionally focused couple therapy. Journal of Couple \& Relationship Therapy. 2014; 13(1):1-24. [DOI:10.1080/1533 2691.2013.836046]

[55] Yoo SH, Matsumoto D, LeRoux JA. The influence of emotion recognition and emotion regulation on intercultural adjustment. International Journal of Intercultural Relations. 2006; 30(3):345-63. [DOI:10.1016/j.ijintrel.2005.08.006] 
[56] Raio CM, Orederu TA, Palazzolo L, Shurick AA, Phelps EA. Cognitive emotion regulation fails the stress test. Proceedings of the National Academy of Sciences. 2013; 110(37):15139-44. [DOI:10.1073/pnas.1305706110] [PMID] [PMCID]

[57] Falconier MK, Jackson JB, Hilpert P, Bodenmann G. Dyadic coping and relationship satisfaction: A meta-analysis. Clinical Psychology Review. 2015; 42:28-46. [DOI:10.1016/j. cpr.2015.07.002] [PMID]

[58] Garnefski N, Baan N, Kraaij V. Psychological distress and cognitive emotion regulation strategies among farmers who fell victim to the foot-and-mouth crisis. Personality and Individual Differences. 2005; 38(6):1317-27. [DOI:10.1016/j. paid.2004.08.014]

[59] Min JA, Yu JJ, Lee CU, Chae JH. Cognitive emotion regulation strategies contributing to resilience in patients with depression and/or anxiety disorders. Comprehensive Psychiatry. 2013; 54(8):1190-7. [DOI:10.1016/j. comppsych.2013.05.008] [PMID]

[60] Desrosiers A, Vine V, Curtiss J, Klemanski DH. Observing nonreactively: A conditional process model linking mindfulness facets, cognitive emotion regulation strategies, and depression and anxiety symptoms. Journal of Affective Disorders. 2014; 165:31-7. [DOI:10.1016/j.jad.2014.04.024] [PMID] 
This Page Intentionally Left Blank 